\title{
Hyperbolic Hemivariational Inequalities for Dynamic Viscoelastic Contact Problems
}

\author{
Anna Kulig
}

Received: 12 December 2011 / Published online: 3 April 2012

(C) The Author(s) 2012. This article is published with open access at Springerlink.com

\begin{abstract}
The paper deals with second order nonlinear evolution inclusions and their applications. First, we study an evolution inclusion involving Volterra-type integral operator which is considered within the framework of an evolution triple of spaces. We provide a result on the unique solvability of the Cauchy problem for the inclusion. Next, we examine a dynamic frictional contact problem of viscoelasticity for materials with long memory and derive a weak formulation of the model in the form of a hemivariational inequality. Then, we embed the hemivariational inequality into a class of second order evolution inclusions involving Volterra-type integral operator and indicate how the result on evolution inclusion is applicable to the model of the contact problem. We conclude with examples of the subdifferential boundary conditions for different types of frictional contact.
\end{abstract}

Keywords Evolution inclusion · Pseudomonotone operator · Volterra-type operator · Multifunction · Hyperbolic · Contact problem · Hemivariational inequality · Viscoelasticity $\cdot$ Clarke subdifferential

Mathematics Subject Classification 35L90 - 35R70 - 45P05 - 47H04 - 47H05 - 74H20 . $74 \mathrm{H} 25$

\section{Introduction}

An important number of problems arising in Mechanics, Physics and Engineering Science lead to mathematical models expressed in terms of nonlinear inclusions and hemivariational inequalities. For this reason the mathematical literature dedicated to this field is extensive and the progress made in the last decades is impressive. It concerns both results on the

This work is based on the author Ph.D. thesis conferred at Jagiellonian University, Krakow, Poland, 2010 .

A. Kulig $(\bowtie)$

Faculty of Mathematics and Computer Science, Institute of Computer Science, Jagiellonian University, ul. Łojasiewicza 6, 30-348 Krakow, Poland

e-mail: anna_k@gazeta.pl 
existence, uniqueness, regularity and behavior of solutions for various classes of nonlinear inclusions as well as results on numerical approach of the solution for the corresponding problems.

The purpose of this paper is to use a recent result on unique solvability of the following second order evolution inclusion

$$
u^{\prime \prime}(t)+A\left(t, u^{\prime}(t)\right)+B(t, u(t))+\int_{0}^{t} C(t-s) u(s) d s+F\left(t, u(t), u^{\prime}(t)\right) \ni f(t)
$$

which is considered on a finite time interval in the framework of evolution triple of spaces $\left(V, H, V^{*}\right)$ and show how the result on the evolution inclusion is applicable to the model of the contact problem. We provide conditions on a unique solvability of the inclusion which were studier earlier in $[18,19]$. Subsequently, we consider the class of evolution hemivariational inequalities of second order of the form

$$
\left\{\begin{array}{r}
\left\langle u^{\prime \prime}(t)+A\left(t, u^{\prime}(t)\right)+B(t, u(t))+\int_{0}^{t} C(t-s) u(s) d s-f(t), v\right\rangle \\
\quad+\int_{\Gamma_{C}} g^{0}\left(x, t, \gamma u(t), \gamma u^{\prime}(t), \gamma u(t), \gamma u^{\prime}(t) ; \gamma v, \gamma v\right) d \Gamma \geq 0 \\
\quad \text { for all } v \in V, \text { a.e. } t \in(0, T), \\
u(0)=u_{0}, u^{\prime}(0)=u_{1},
\end{array}\right.
$$

where $g^{0}$ denotes the generalized directional derivative of Clarke of a possibly nonconvex function $g, \gamma$ is a trace map and $\langle\cdot, \cdot\rangle$ stands for the duality pairing between $V^{*}$ and $V$.

Our study includes the modeling of a mechanical problem and its variational analysis. We derive the hemivariational inequality for the displacement field from nonconvex superpotentials through the generalized Clarke subdifferential. The novelty of the model is to deal with nonlinear elasticity and viscosity operators and to consider the coupling between two kinds of nonmonotone possibly multivalued boundary conditions which depend on the normal (respectively, tangential) components of both the displacement and velocity. We recall that the notion of hemivariational inequality is based on the generalized gradient of Clarke [6] and has been introduced in the early 1980s by Panagiotopoulos [33, 34]. We also note that the existence of solutions to the second order evolution inclusions as well as to the corresponding dynamic hemivariational inequalities has been studied, for instance, in Migórski [22-25], Migórski and Ochal [27, 28] and Kulig [17, 18].

Finally, in order to illustrate the cross fertilization between rigorous mathematical description and Nonlinear Analysis on one hand, and modeling and applications on the other hand, we provide several examples of contact and friction subdifferential boundary conditions.

The paper is structured as follows. In Sect. 2 we recall some preliminary material. In Sect. 3 we describe the dynamic viscoelastic contact problem and present its classical and weak formulation. Next, we recall a result on the existence and uniqueness of solutions to the Cauchy problem for the second order nonlinear evolution inclusion involving a Volterratype integral operator. We establish the link between a nonlinear evolution inclusion and the hemivariational inequality, and apply the aforementioned results to the viscoelastic contact problem with a memory term. The review of several examples of contact and friction subdifferential boundary conditions which illustrates the applicability of our results is provided in Sect. 4. 


\section{Preliminaries}

In this section we recall the notation and basic definitions needed in the sequel.

Given a Banach space $\left(X,\|\cdot\|_{X}\right)$, we use the symbol $w$ - $X$ to denote the space $X$ endowed with the weak topology. The class of linear and bounded operators from $X$ to $X^{*}$ is denoted by $\mathcal{L}\left(X, X^{*}\right)$. If $U \subset X$, then we have $\|U\|_{X}=\sup \left\{\|x\|_{X}: x \in U\right\}$. The duality between $X$ and its dual is denoted by $\langle\cdot, \cdot\rangle_{X^{*} \times X}$.

The linear space of second order symmetric tensors on $\mathbb{R}^{d}$ will be denoted by $\mathbb{S}^{d}$. The inner product and the corresponding norm on $\mathbb{S}^{d}$ is defined similarly to the inner product on $\mathbb{R}^{d}$, i.e.,

$$
\begin{aligned}
& u \cdot v=u_{i} v_{i}, \quad\|v\|_{\mathbb{R}^{d}}=(v \cdot v)^{1 / 2} \quad \text { for all } u, v \in \mathbb{R}^{d}, \\
& \sigma: \tau=\sigma_{i j} \tau_{i j}, \quad\|\tau\|_{\mathbb{S}^{d}}=(\tau: \tau)^{1 / 2} \text { for all } \sigma, \tau \in \mathbb{S}^{d} .
\end{aligned}
$$

The summation convention over repeated indices is used here and below.

Let $\Omega \subset \mathbb{R}^{d}$ be a bounded domain with Lipschitz boundary $\Gamma$. In what follows we will need the following Hilbert spaces with their inner products

$$
\begin{array}{ll}
H=L^{2}\left(\Omega ; \mathbb{R}^{d}\right) & \langle u, v\rangle_{H}=\int_{\Omega} u \cdot v d x, \\
\mathcal{H}=\left\{\tau=\left\{\tau_{i j}\right\} \mid \tau_{i j}=\tau_{j i} \in L^{2}(\Omega)\right\} & \langle\sigma, \tau\rangle_{\mathcal{H}}=\int_{\Omega} \sigma: \tau d x, \\
H_{1}=\{u \in H \mid \varepsilon(u) \in \mathcal{H}\} & \langle u, v\rangle_{H_{1}}=\langle u, v\rangle_{H}+\langle\varepsilon(u), \varepsilon(v)\rangle_{\mathcal{H}}, \\
\mathcal{H}_{1}=\{\tau \in \mathcal{H} \mid \operatorname{Div} \tau \in H\} & \langle\sigma, \tau\rangle_{\mathcal{H}_{1}}=\langle\sigma, \tau\rangle_{\mathcal{H}}+\langle\operatorname{Div} \sigma, \operatorname{Div} \tau\rangle_{H} .
\end{array}
$$

Here $\varepsilon: H^{1}\left(\Omega ; \mathbb{R}^{d}\right) \rightarrow L^{2}\left(\Omega ; \mathbb{S}^{d}\right)$ denotes the deformation operator, Div: $\mathcal{H}_{1} \rightarrow L^{2}\left(\Omega ; \mathbb{R}^{d}\right)$ stands for the divergence operator, where

$$
\varepsilon(u)=\left\{\varepsilon_{i j}(u)\right\}, \quad \varepsilon_{i j}(u)=\frac{1}{2}\left(u_{i, j}+u_{j, i}\right), \quad \operatorname{Div} \sigma=\left\{\sigma_{i j, j}\right\} .
$$

The index following comma indicates a partial derivative.

Given $v \in H_{1}$ we denote its trace $\gamma v$ on $\Gamma$ by $v$, where $\gamma: H^{1}\left(\Gamma ; \mathbb{R}^{d}\right) \rightarrow H^{1 / 2}\left(\Gamma ; \mathbb{R}^{d}\right) \subset$ $L^{2}\left(\Gamma ; \mathbb{R}^{d}\right)$ is the trace map. Let $n$ denote the outward unit normal vector to $\Gamma$. Since $\Gamma$ is Lipschitz continuous, the normal vector is defined a.e. on $\Gamma$. For $v \in H^{1 / 2}\left(\Gamma ; \mathbb{R}^{d}\right)$ we denote its normal and tangential components by $v_{N}=v \cdot n$ and $v_{T}=v-v_{N} n$.

Let $V$ be a separable Banach space. We identify $H$ with its dual and we consider a Gelfand triple $V \subset H \subset V^{*}$ where all embeddings are compact, dense and continuous (see $[7,44])$. We will need the following spaces $\mathcal{V}=L^{2}(0, T ; V)$ and $\mathcal{W}=\left\{w \in \mathcal{V}: w^{\prime} \in \mathcal{V}^{*}\right\}$. The time derivative involved in the definition of $\mathcal{W}$ is understood in the sense of vector valued distributions. It is well known that $\mathcal{W}$ is a separable Banach space equipped with the norm $\|v\|_{\mathcal{W}}=\|v\|_{\mathcal{V}}+\|v\|_{\mathcal{V}^{*}}$ and $\mathcal{W} \subset \mathcal{V} \subset L^{2}(0, T ; H) \subset \mathcal{V}^{*}$

Let us recall some definitions needed in the next sections.

Measurable multifunction Let $(\Omega, \Sigma)$ be a measurable space, $\mathcal{X}$ be a separable Banach space and $F: \Omega \rightarrow 2^{\mathcal{X}}$. The multifunction $F$ is said to be measurable if for every $U \subset X$ open, we have $F^{-}(U)=\{\omega \in \Omega: F(\omega) \cap U \neq \emptyset\} \in \Sigma$. 
Generalized directional derivative Let $X$ be a Banach space. The generalized directional derivative of Clarke of locally Lipschitz function $h: X \rightarrow \mathbb{R}$ at $x \in X$ in the direction $v \in X$, denoted by $h^{0}(x ; v)$, is defined by (cf. [6])

$$
h^{0}(x ; v)=\limsup _{y \rightarrow x, \lambda \downarrow 0} \frac{h(y+\lambda v)-h(y)}{\lambda} .
$$

Generalized gradient Let $X$ be a Banach space. The generalized gradient of a function $h: X \rightarrow \mathbb{R}$ at $x \in X$, denoted by $\partial h(x)$, is a subset of a dual space $X^{*}$ given by $\partial h(x)=$ $\left\{\zeta \in X^{*} \mid h^{0}(x ; v) \geq\langle\zeta, v\rangle_{X^{*} \times X}\right.$ for all $\left.v \in X\right\}$.

Regular function A locally Lipschitz function $h$ is called regular (in the sense of Clarke) at $x \in X$ if for all $v \in X$ the one-sided directional derivative $h^{\prime}(x ; v)$ exists and satisfies $h^{0}(x ; v)=h^{\prime}(x ; v)$ for all $v \in X$.

Finally we state results needed in a sequel whose proofs can be found in Kulig [18].

Lemma 1 Let $X$ and $Y$ be Banach spaces and $\varphi: X \times Y \rightarrow \mathbb{R}$ be such that

(i) $\varphi(\cdot, y)$ is continuous for all $y \in Y$;

(ii) $\varphi(x, \cdot)$ is locally Lipschitz on $Y$ for all $x \in X$;

(iii) there is a constant $c>0$ such that for all $\eta \in \partial \varphi(x, y)$, we have

$$
\|\eta\|_{Y^{*}} \leq c\left(1+\|x\|_{X}+\|y\|_{Y}\right) \quad \text { for all } x \in X, y \in Y,
$$

where $\partial \varphi$ denotes the generalized gradient of $\varphi(x, \cdot)$.

Then $\varphi$ is continuous on $X \times Y$.

Proposition 2 Let $X$ be a separable reflexive Banach space, $0<T<\infty$ and $\varphi:(0, T) \times$ $X \rightarrow \mathbb{R}$ be a function such that $\varphi(\cdot, x)$ is measurable for all $x \in X$ and $\varphi(t, \cdot)$ is locally Lipschitz for all $t \in(0, T)$. Then the multifunction $(0, T) \times X \ni(t, x) \mapsto \partial \varphi(t, x) \subset X^{*}$ is measurable, where $\partial \varphi$ denotes the Clarke generalized gradient of $\varphi(t, \cdot)$.

\section{Dynamic Viscoelastic Contact Problem with Memory Term}

In this section we present a short description of the modeled process, give its weak formulation which is a hyperbolic hemivariational inequality and obtain results on existence and uniqueness of weak solutions.

\subsection{Physical Setting of the Problem}

The physical setting and the process are as follows. The set $\Omega$ is occupied by a viscoelastic body in $\mathbb{R}^{d}$ ( $d=2,3$ in applications) which is referred to as the reference configuration. We assume that $\Omega$ is a bounded domain with Lipschitz boundary $\Gamma$ which is divided into three mutually disjoint measurable parts $\Gamma_{D}, \Gamma_{N}$ and $\Gamma_{C}$ with $m\left(\Gamma_{D}\right)>0$.

We study the process of evolution of the mechanical state in time interval $[0, T], 0<T<$ $\infty$. The system evolves in time as a result of applied volume forces and surface tractions. The description of this evolution is done by introducing a vector function $u=u(x, t)=$ $\left(u_{1}(x, t), \ldots, u_{d}(x, t)\right)$ which describes the displacement at time $t$ of a particle that has 
the position $x=\left(x_{1}, \ldots, x_{d}\right)$ in the reference configuration. We denote by $\sigma=\sigma(x, t)=$ $\left(\sigma_{i j}(x, t)\right)$ the stress tensor and by $\varepsilon(u)=\left(\varepsilon_{i j}(u)\right)$ the linearized (small) strain tensor whose components are given by (a compatibility condition) $\varepsilon_{i j}=\varepsilon_{i j}(u)=\frac{1}{2}\left(u_{i, j}+u_{j, i}\right)$, where $i$, $j=1, \ldots, d$. In cases where an index appears twice, we use the summation convention. We also put $Q=\Omega \times(0, T)$.

Since the process is dynamic, we deal with the dynamic equation of motion representing momentum conservation (cf. $[11,33]$ ) and governing the evolution of the state of the body

$$
u^{\prime \prime}(t)-\operatorname{Div} \sigma(t)=f_{0}(t) \text { in } Q,
$$

where Div denotes the divergence operator for tensor valued functions and $f_{0}$ is the density of applied volume forces such as gravity. We assume that the mass density is constant and set equal to one.

In the model the material is assumed to be viscoelastic and for its description we suppose a general constitutive law of the form

$$
\sigma(t)=\mathcal{A}\left(t, \varepsilon\left(u^{\prime}(t)\right)\right)+\mathcal{B}(t, \varepsilon(u(t)))+\int_{0}^{t} \mathcal{C}(t-s) \varepsilon(u(s)) d s \quad \text { in } Q .
$$

Here $\mathcal{A}$ is a nonlinear operator describing the purely viscous properties of the material while $\mathcal{B}$ and $\mathcal{C}$ are the nonlinear elasticity and the linear relaxation operators, respectively. Note that the operators $\mathcal{A}$ and $\mathcal{B}$ may depend explicitly on the time variable and this is the case when the viscosity and elasticity properties of the material depend on the temperature field which plays the role of a parameter and whose evolution in time is prescribed. When $\mathcal{C}=0$ the constitutive law (1) reduces to a viscoelastic constitutive law (the so called Kelvin-Voigt law) with short memory and in the case when $\mathcal{A}=0$, it reduces to an elastic constitutive law with long memory.

Next, we describe the boundary conditions. The body is supposed to be held fixed on the part $\Gamma_{D}$ of the surface, so the displacement $u=0$ on $\Gamma_{D} \times(0, T)$. On the part $\Gamma_{N}$ a prescribed surface force (traction) $f_{1}=f_{1}(x, t)$ is applied, thus we have the condition $\sigma(t) v=f_{1}$ on $\Gamma_{N} \times(0, T)$. Here $v \in \mathbb{R}^{d}$ denotes the outward unit normal to $\Gamma$ and $\sigma(t) v$ represents the boundary stress vector. The body may come in contact over the part $\Gamma_{C}$ of its surface. As it is met in the literature (cf. [10, 11, 41, 42]) the conditions on the contact surface are naturally divided to conditions in the normal direction and those in the tangential direction, cf. Sect. 5.4 of [11] for the normal approach and the tangential process. In the model under consideration, the frictional contact on the part $\Gamma_{C}$ is described by the subdifferential boundary conditions of the form

$$
\begin{aligned}
& -\sigma_{v}(t) \in \partial j_{1}\left(x, t, u(t), u^{\prime}(t), u_{v}(t)\right)+\partial j_{2}\left(x, t, u(t), u^{\prime}(t), u_{v}^{\prime}(t)\right) \\
& -\sigma_{\tau}(t) \in \partial j_{3}\left(x, t, u(t), u^{\prime}(t), u_{\tau}(t)\right)+\partial j_{4}\left(x, t, u(t), u^{\prime}(t), u_{\tau}^{\prime}(t)\right)
\end{aligned}
$$

on $\Gamma_{C} \times(0, T)$, where $\sigma_{v}$ and $\sigma_{\tau}, u_{v}$ and $u_{\tau}, u_{v}^{\prime}$ and $u_{\tau}^{\prime}$ denote the normal and the tangential components of the stress tensor, the displacement and the velocity, respectively. The functions $j_{k}, k=1, \ldots, 4$ are prescribed and locally Lipschitz in their last variables. The component $\sigma_{\tau}$ represents the friction force on the contact surface and $\partial j_{k}, k=1, \ldots, 4$ denote the Clarke subdifferentials of the superpotentials $j_{k}, k=1, \ldots, 4$ with respect to their last variables. Concrete examples of contact models which lead to aforementioned subdifferential boundary conditions will be provided in Sect. 4 .

Finally, we prescribe the initial conditions for the displacement and the velocity, i.e.,

$$
u(0)=u_{0} \quad \text { and } \quad u^{\prime}(0)=u_{1} \quad \text { in } \Omega,
$$


where $u_{0}$ and $u_{1}$ denote the initial displacement and the initial velocity, respectively. In what follows we skip occasionally the dependence of various functions on the spatial variable $x \in \Omega \cup \Gamma$.

Collecting the equations and conditions described above, we obtain the following formulation of the mechanical problem: find a displacement field $u: Q \rightarrow \mathbb{R}^{d}$ and a stress field $\sigma: Q \rightarrow \mathcal{S}_{d}$ such that

$$
\begin{array}{ll}
u^{\prime \prime}(t)-\operatorname{Div} \sigma(t)=f_{0}(t) & \text { in } Q, \\
\sigma(t)=\mathcal{A}\left(t, \varepsilon\left(u^{\prime}(t)\right)\right)+\mathcal{B}(t, \varepsilon(u(t)))+\int_{0}^{t} \mathcal{C}(t-s) \varepsilon(u(s)) d s & \text { in } Q, \\
u(t)=0 & \text { on } \Gamma_{D} \times(0, T), \\
\sigma(t) v=f_{1} & \text { on } \Gamma_{N} \times(0, T), \\
-\sigma_{\nu}(t) \in \partial j_{1}\left(t, u(t), u^{\prime}(t), u_{v}(t)\right)+\partial j_{2}\left(t, u(t), u^{\prime}(t), u_{v}^{\prime}(t)\right) & \text { on } \Gamma_{C} \times(0, T), \\
-\sigma_{\tau}(t) \in \partial j_{3}\left(t, u(t), u^{\prime}(t), u_{\tau}(t)\right)+\partial j_{4}\left(t, u(t), u^{\prime}(t), u_{\tau}^{\prime}(t)\right) & \text { on } \Gamma_{C} \times(0, T), \\
u(0)=u_{0}, \quad u^{\prime}(0)=u_{1} & \text { in } \Omega .
\end{array}
$$

The problem above represents the classical formulation of the viscoelastic frictional contact problem. The conditions (6) and (7) introduce one of the main difficulties to the problem since the superpotentials are nonconvex and nonsmooth in general. This is the reason why the problem (2)-(8) has no classical solutions, i.e., solutions which posses all necessary classical derivatives and satisfy the relations in the usual sense at each point and at each time instant. In the following we formulate the above problem in a weak sense.

\subsection{Weak Formulation of the Problem}

In this section we give a weak formulation of the classical viscoelastic frictional contact problem (2)-(8). Due to the Clarke subdifferential boundary conditions (6) and (7) this formulation will be a hyperbolic hemivariational inequality. We introduce

$$
V=\left\{v \in H_{1} \mid v=0 \text { on } \Gamma_{D}\right\} .
$$

This is a closed subspace of $H_{1}$ and so it is a Hilbert space with the inner product and the corresponding norm given by $(u, v)_{V}=(\varepsilon(u), \varepsilon(v))_{\mathcal{H}}$ and $\|v\|=\|\varepsilon(v)\|_{\mathcal{H}}$ for $u, v \in V$. By the Korn inequality $\|v\|_{H_{1}} \leq c\|\varepsilon(v)\|_{\mathcal{H}}$ for $v \in V$ with $c>0$ (cf. Sect. 6.3 of [31]), it follows that $\|\cdot\|_{H_{1}}$ and $\|\cdot\|$ are the equivalent norms on $V$. Identifying $H=L^{2}\left(\Omega ; \mathbb{R}^{d}\right)$ with its dual, we have an evolution triple of spaces $\left(V, H, V^{*}\right)$ with dense, continuous and compact embeddings. For this evolution triple, analogously as in Sect. 2, we define the spaces $\mathcal{V}=L^{2}(0, T ; V), \widehat{\mathcal{H}}=L^{2}(0, T ; H), \mathcal{V}^{*}=L^{2}\left(0, T ; V^{*}\right)$ and $\mathcal{W}=\left\{v \in \mathcal{V} \mid v^{\prime} \in \mathcal{V}^{*}\right\}$. The duality pairing between $V^{*}$ and $V$ is denoted by $\langle\cdot, \cdot\rangle$.

We admit the following hypotheses on the data of the problem (2)-(8).

$\underline{H(\mathcal{A})}$ : The viscosity operator $\mathcal{A}: Q \times \mathbb{S}^{d} \rightarrow \mathbb{S}^{d}$ is such that

(i) $\mathcal{A}(\cdot, \cdot, \varepsilon)$ is measurable on $Q$ for all $\varepsilon \in \mathbb{S}^{d}$;

(ii) $\mathcal{A}(x, t, \cdot)$ is continuous on $\mathbb{S}^{d}$ for a.e. $(x, t) \in Q$; 
(iii) $\|\mathcal{A}(x, t, \varepsilon)\|_{\mathbb{S}^{d}} \leq \tilde{a_{1}}(x, t)+\tilde{a_{2}}\|\varepsilon\|_{\mathbb{S}^{d}}$ for all $\varepsilon \in \mathbb{S}^{d}$, a.e. $(x, t) \in Q$ with $\widetilde{a_{1}} \in L^{2}(Q)$, $\widetilde{a_{1}}, \widetilde{a_{2}} \geq 0$

(iv) $\left(\mathcal{A}\left(x, t, \varepsilon_{1}\right)-\mathcal{A}\left(x, t, \varepsilon_{2}\right)\right):\left(\varepsilon_{1}-\varepsilon_{2}\right) \geq \tilde{a}_{4}\left\|\varepsilon_{1}-\varepsilon_{2}\right\|_{\mathbb{S}^{d}}^{2}$ for all $\varepsilon_{1}, \varepsilon_{2} \in \mathbb{S}^{d}$, a.e. $(x, t) \in Q$ with $\widetilde{a_{4}}>0$

(v) $\mathcal{A}(x, t, \varepsilon): \varepsilon \geq \widetilde{a_{3}}\|\varepsilon\|_{\mathbb{S}^{d}}^{2}$ for all $\varepsilon \in \mathbb{S}^{d}$, a.e. $(x, t) \in Q$ with $\widetilde{a_{3}}>0$.

Remark 3 It should be remarked that the growth condition $H(\mathcal{A})($ iii) excludes terms with power greater than one, but is satisfied within linearized viscoelasticity, and is satisfied by truncated operators, cf. [11, 41].

$H(\mathcal{B})$ : The elasticity operator $\mathcal{B}: Q \times \mathbb{S}^{d} \rightarrow \mathbb{S}^{d}$ is such that

(i) $\mathcal{B}(\cdot, \cdot, \varepsilon)$ is measurable on $Q$ for all $\varepsilon \in S_{d}$;

(ii) $\|\mathcal{B}(x, t, \varepsilon)\|_{\mathbb{S}^{d}} \leq \widetilde{b_{1}}(x, t)+\widetilde{b_{2}}\|\varepsilon\|_{\mathbb{S}^{d}}$ for all $\varepsilon \in \mathbb{S}^{d}$, a.e. $(x, t) \in Q$ with $\widetilde{b_{1}} \in L^{2}(Q), \widetilde{b_{1}}$, $\widetilde{b_{2}} \geq 0$

(iii) $\left\|\mathcal{B}\left(x, t, \varepsilon_{1}\right)-\mathcal{B}\left(x, t, \varepsilon_{2}\right)\right\|_{\mathbb{S}^{d}} \leq L_{\mathcal{B}}\left\|\varepsilon_{1}-\varepsilon_{2}\right\|_{\mathbb{S}^{d}}$ for all $\varepsilon_{1}, \varepsilon_{2} \in \mathbb{S}^{d}$, a.e. $(x, t) \in Q$ with $L_{\mathcal{B}}>0$.

Remark 4 If $\mathcal{B}(x, t, \cdot) \in \mathcal{L}\left(\mathbb{S}^{d}, \mathbb{S}^{d}\right)$ for a.e. $(x, t) \in Q$, the conditions $H(\mathcal{B})$ (ii) and (iii) hold. Thus the hypothesis $H(\mathcal{B})$ is more general than the ones considered in [23-27, 32, 35] where the elasticity operator is assumed to be linear (which corresponds to the Hooke law).

$H(\mathcal{C})$ : The relaxation operator $\mathcal{C}: Q \times \mathbb{S}^{d} \rightarrow \mathbb{S}^{d}$ is of the form $\mathcal{C}(x, t, \varepsilon)=c(x, t) \varepsilon$ and $\overline{c(x, t)}=\left\{c_{i j k l}(x, t)\right\}$ with $c_{i j k l}=c_{j i k l}=c_{l k i j} \in L^{\infty}(Q)$.

$\underline{H(f)}: f_{0} \in L^{2}(0, T ; H), f_{1} \in L^{2}\left(0, T ; L^{2}\left(\Gamma_{N} ; \mathbb{R}^{d}\right)\right), u_{0} \in V, u_{1} \in H$.

The functions $j_{k}$ for $k=1,2$ satisfy the following

$\underline{H\left(j_{k}\right)}$ : The function $j_{k}: \Gamma_{C} \times(0, T) \times\left(\mathbb{R}^{d}\right)^{2} \times \mathbb{R} \rightarrow \mathbb{R}$ is such that

(i) $j_{k}(\cdot, \cdot, \zeta, \rho, r)$ is measurable for all $\zeta, \rho \in \mathbb{R}^{d}, r \in \mathbb{R}$, $j_{k}(\cdot, \cdot, v(\cdot), w(\cdot), 0) \in L^{1}\left(\Gamma_{C} \times(0, T)\right)$ for all $v, w \in L^{2}\left(\Gamma_{C} ; \mathbb{R}^{d}\right) ;$

(ii) $j_{k}(x, t, \cdot, \cdot, r)$ is continuous for all $r \in \mathbb{R}$, a.e. $(x, t) \in \Gamma_{C} \times(0, T)$, $j_{k}(x, t, \zeta, \rho, \cdot)$ is locally Lipschitz for all $\zeta, \rho \in \mathbb{R}^{d}$, a.e. $(x, t) \in \Gamma_{C} \times(0, T)$;

(iii) $\left|\partial j_{k}(x, t, \zeta, \rho, r)\right| \leq c_{k 0}+c_{k 1}\|\zeta\|+c_{k 2}\|\rho\|+c_{k 3}|r|$ for all $\zeta, \rho \in \mathbb{R}^{d}, r \in \mathbb{R}$, a.e. $(x, t) \in \Gamma_{C} \times(0, T)$ with $c_{k j} \geq 0, j=0,1,2,3$, where $\partial j_{k}$ denotes the Clarke subdifferential of $j_{k}(x, t, \zeta, \rho, \cdot)$;

(iv) $j_{k}^{0}(x, t, \cdot, \cdot, \cdot ; s)$ is upper semicontinuous on $\left(\mathbb{R}^{d}\right)^{2} \times \mathbb{R}$ for all $s \in \mathbb{R}$, a.e. $(x, t) \in$ $\Gamma_{C} \times(0, T)$, where $j_{k}^{0}$ denotes the generalized directional derivative of Clarke of $j_{k}(x, t, \zeta, \rho, \cdot)$ in the direction $s$.

The functions $j_{k}$ for $k=3,4$ satisfy the corresponding conditions with the last variable being in $\mathbb{R}^{d}$.

Moreover, we need the following hypothesis.

$H(j)_{\text {reg }}$ : The functions $j_{k}$ for $k=1, \ldots, 4$ are such that for all $\zeta, \rho \in \mathbb{R}^{d}$, a.e. $(x, t) \in$ $\Gamma_{C} \times(0, T)$, either all $j_{k}(x, t, \zeta, \rho, \cdot)$ are regular or all $-j_{k}(x, t, \zeta, \rho, \cdot)$ are regular for $k=1, \ldots, 4$.

The above hypotheses are realistic with respect to the physical data and the process modeling. We will see this in the specific examples of contact laws which are given in Sect. 4 . 
Next, let $v \in V$. We define $f \in \mathcal{V}^{*}$ by

$$
\langle f(t), v\rangle_{V^{*} \times V}=\left\langle f_{0}(t), v\right\rangle_{H}+\left\langle f_{1}(t), v\right\rangle_{L^{2}\left(\Gamma_{N} ; \mathbb{R}^{d}\right)}
$$

for a.e. $t \in(0, T)$. Assuming that the functions in the problem (2)-(8) are sufficiently regular, using the equation of motion (2) and the Green formula (cf. [44]), we obtain

$$
\left\langle u^{\prime \prime}(t), v\right\rangle+\langle\sigma(t), \varepsilon(v)\rangle_{\mathcal{H}}-\int_{\Gamma} \sigma(t) v \cdot v(x) d \Gamma=\left\langle f_{0}(t), v\right\rangle_{H}
$$

for a.e. $t \in(0, T)$. From the boundary conditions (4) and (5), we have

$$
\int_{\Gamma} \sigma(t) v \cdot v d \Gamma=\int_{\Gamma_{N}} f_{1}(t) \cdot v d \Gamma+\int_{\Gamma_{C}}\left(\sigma_{\tau}(t) \cdot v_{\tau}+\sigma_{v}(t) v_{v}\right) d \Gamma .
$$

On the other hand, the subdifferential boundary conditions (6) and (7) imply

$$
\begin{aligned}
-\sigma_{v}(t) r \leq j_{1}^{0}\left(x, t, u(t), u^{\prime}(t), u_{v}(t) ; r\right)+j_{2}^{0}\left(x, t, u(t), u^{\prime}(t), u_{v}^{\prime}(t) ; r\right) & \text { for all } r \in \mathbb{R}, \\
-\sigma_{\tau}(t) \cdot \xi \leq j_{3}^{0}\left(x, t, u(t), u^{\prime}(t), u_{\tau}(t) ; \xi\right)+j_{4}^{0}\left(x, t, u(t), u^{\prime}(t), u_{\tau}^{\prime}(t) ; \xi\right) & \text { for all } \xi \in \mathbb{R}^{d} .
\end{aligned}
$$

Using the constitutive law (3) and the above relations, we obtain the following weak formulation of the problem (2)-(8) which is called hemivariational inequality.

Problem (HVI): find $u:(0, T) \rightarrow V$ such that $u \in \mathcal{V}, u^{\prime} \in \mathcal{W}$ and

$$
\left\{\begin{array}{c}
\left\langle u^{\prime \prime}(t), v\right\rangle+\left\langle\mathcal{A}\left(t, \varepsilon\left(u^{\prime}(t)\right)\right)+\mathcal{B}(t, \varepsilon(u(t)))+\int_{0}^{t} \mathcal{C}(t-s) \varepsilon(u(s)) d s, \varepsilon(v)\right\rangle_{\mathcal{H}} \\
\quad+\int_{\Gamma_{C}}\left(j_{1}^{0}\left(x, t, u(t), u^{\prime}(t), u_{v}(t) ; v_{v}\right)+j_{2}^{0}\left(x, t, u(t), u^{\prime}(t), u_{v}^{\prime}(t) ; v_{v}\right)\right. \\
\left.\quad+j_{3}^{0}\left(x, t, u(t), u^{\prime}(t), u_{\tau}(t) ; v_{\tau}\right)+j_{4}^{0}\left(x, t, u(t), u^{\prime}(t), u_{\tau}^{\prime}(t) ; v_{\tau}\right)\right) d \Gamma \\
\geq\langle f(t), v\rangle \quad \text { for all } v \in V, \text { a.e. } t \in(0, T), \\
u(0)=u_{0}, \quad u^{\prime}(0)=u_{1} .
\end{array}\right.
$$

3.3 Evolution Inclusion for Hemivariational Inequality

In this section we state a result on the existence of solutions to second order evolution inclusions and apply it to an abstract hemivariational inequality. To this end, let $Z=H^{\delta}\left(\Omega ; \mathbb{R}^{d}\right)$, $\delta \in(1 / 2,1)$ and $\gamma: Z \rightarrow L^{2}\left(\Gamma_{C} ; \mathbb{R}^{d}\right)$ be the trace operator. Let $\gamma^{*}: L^{2}\left(\Gamma_{C} ; \mathbb{R}^{d}\right) \rightarrow Z^{*}$ stand for the adjoint operator to $\gamma$.

Problem $\mathcal{P}$ : find $u \in \mathcal{V}$ such that $u^{\prime} \in \mathcal{W}$ and

$$
\left\{\begin{array}{l}
u^{\prime \prime}(t)+A\left(t, u^{\prime}(t)\right)+B(t, u(t))+\int_{0}^{t} C(t-s) u(s) d s \\
\quad+F\left(t, u(t), u^{\prime}(t)\right) \ni f(t) \quad \text { a.e. } t \in(0, T) \\
u(0)=u_{0}, \quad u^{\prime}(0)=u_{1}
\end{array}\right.
$$

where $A, B:(0, T) \times V \rightarrow V^{*}$ are nonlinear operators, $C(t)$ is a bounded linear operator for a.e. $t \in(0, T)$ and $F:(0, T) \times V \times V \rightarrow 2^{Z^{*}}$ is a multivalued mapping. Let us notice that the initial conditions in Problem $\mathcal{P}$ have sense in $V$ and $H$ since the embeddings $\{v \in$ $\left.\mathcal{V} \mid v^{\prime} \in \mathcal{W}\right\} \subset C(0, T ; V)$ and $\mathcal{W} \subset C(0, T ; H)$ are continuous (see [7, 44]). A solution to Problem $\mathcal{P}$ is understood as follows. 
Definition 5 A function $u \in \mathcal{V}$ is a solution of Problem $\mathcal{P}$ if and only if $u^{\prime} \in \mathcal{W}$ and there exists $z \in \mathcal{Z}^{*}$ such that

$$
\left\{\begin{array}{l}
u^{\prime \prime}(t)+A\left(t, u^{\prime}(t)\right)+B(t, u(t))+\int_{0}^{t} C(t-s) u(s) d s+z(t)=f(t) \quad \text { a.e. } t \in(0, T), \\
z(t) \in F\left(t, u(t), u^{\prime}(t)\right) \quad \text { a.e. } t \in(0, T) \\
u(0)=u_{0}, \quad u^{\prime}(0)=u_{1} .
\end{array}\right.
$$

We will need the following hypotheses on the data.

$H(A)$ : The operator $A:(0, T) \times V \rightarrow V^{*}$ is such that

(i) $A(\cdot, v)$ is measurable on $(0, T)$ for all $v \in V$;

(ii) $A(t, \cdot)$ is strongly monotone for a.e. $t \in(0, T)$, i.e. there exists $m_{1}>0$ such that $\langle A(t, v)-A(t, u), v-u\rangle \geq m_{1}\|v-u\|^{2}$ for all $u, v \in V$, a.e. $t \in(0, T)$

(iii) $\|A(t, v)\|_{V^{*}} \leq a_{0}(t)+a_{1}\|v\|$ for all $v \in V$, a.e. $t \in(0, T)$ with $a_{0} \in L^{2}(0, T), a_{0} \geq 0$ and $a_{1}>0$

(iv) $\langle A(t, v), v\rangle \geq \alpha\|v\|^{2}$ for all $v \in V$, a.e. $t \in(0, T)$ with $\alpha>0$;

(v) $A(t, \cdot)$ is hemicontinuous for a.e. $t \in(0, T)$.

Remark 6 The hypothesis $H(A)$ implies the operator $A$ is pseudomonotone. Indeed, strong monotonicity clearly implies monotonicity which with hemicontinuity entails (cf. Proposition 27.6(a), p. 586, of Zeidler [44]) pseudomonotonicity. We also recall (cf. Remark 1.1.13 of [8]) that for monotone operators, demicontinuity and hemicontinuity are equivalent notions.

$\underline{H(B)}$ : The operator $B:(0, T) \times V \rightarrow V^{*}$ is such that

(i) $B(\cdot, v)$ is measurable on $(0, T)$ for all $v \in V$;

(ii) $B(t, \cdot)$ is Lipschitz continuous for a.e. $t \in(0, T)$, i.e. $\|B(t, u)-B(t, v)\|_{V^{*}} \leq L_{B} \| u-$ $v \|$ for all $u, v \in V$, a.e. $t \in(0, T)$ with $L_{B}>0$;

(iii) $\|B(t, v)\|_{V^{*}} \leq b_{0}(t)+b_{1}\|v\|$ for all $v \in V$, a.e. $t \in(0, T)$ with $b_{0} \in L^{2}(0, T)$ and $b_{0}$, $b_{1} \geq 0$.

$\underline{H(C)}$ : The operator $C$ satisfies $C \in L^{2}\left(0, T ; \mathcal{L}\left(V, V^{*}\right)\right)$.

$\underline{H(F)}$ : The multifunction $F:(0, T) \times V \times V \rightarrow \mathcal{P}_{f c}\left(Z^{*}\right)$ is such that

(i) $F(\cdot, u, v)$ is measurable on $(0, T)$ for all $u, v \in V$;

(ii) $F(t, \cdot, \cdot)$ is upper semicontinuous from $V \times V$ into $w$-Z* for a.e. $t \in(0, T)$, where $V \times V$ is endowed with $(Z \times Z)$-topology;

(iii) $\|F(t, u, v)\|_{Z^{*}} \leq d_{0}(t)+d_{1}\|u\|+d_{2}\|v\|$ for all $u, v \in V$, a.e. $t \in(0, T)$ with $d_{0} \in$ $L^{2}(0, T)$ and $d_{0}, d_{1}, d_{2} \geq 0$.

$\underline{H(F)_{1}}$ : The multifunction $F:(0, T) \times V \times V \rightarrow \mathcal{P}_{f c}\left(Z^{*}\right)$ satisfies $H(F)$ and

(iv) $\left\langle F\left(t, u_{1}, v_{1}\right)-F\left(t, u_{2}, v_{2}\right), v_{1}-v_{2}\right\rangle_{Z^{*} \times Z} \geq-m_{2}\left\|v_{1}-v_{2}\right\|^{2}-m_{3}\left\|v_{1}-v_{2}\right\|\left\|u_{1}-u_{2}\right\|$ for all $u_{i}, v_{i} \in V, i=1,2$, a.e. $t \in(0, T)$ with $m_{2}, m_{3} \geq 0$.

$\underline{\left(H_{0}\right)}: f \in \mathcal{V}^{*}, u_{0} \in V, u_{1} \in H$.

$\left(H_{1}\right): \alpha>2 \sqrt{3} c_{e}\left(d_{1} T+d_{2}\right)$, where $c_{e}>0$ is the embedding constant of $V$ into $Z$, i.e., $\overline{\|\cdot\|_{Z}} \leq c_{e}\|\cdot\|$.

$\underline{\left(H_{2}\right)}: m_{1}>m_{2}+\frac{1}{\sqrt{2}} m_{3} T$. 
Theorem 7 Under the hypotheses $H(A), H(B), H(C), H(F)_{1},\left(H_{0}\right),\left(H_{1}\right)$ and $\left(H_{2}\right)$, Problem $\mathcal{P}$ admits a unique solution.

For the proof, we refer to Theorem 8 of [19].

We are now in a position to apply Theorem 7 to the hemivariational inequality problem we are dealing with. We define the following operators $A, B, C:(0, T) \times V \rightarrow V^{*}$ by

$$
\begin{aligned}
& \langle A(t, u), v\rangle=(\mathcal{A}(x, t, \varepsilon(u)), \varepsilon(v))_{\mathcal{H}}, \\
& \langle B(t, u), v\rangle=(\mathcal{B}(x, t, \varepsilon(u)), \varepsilon(v))_{\mathcal{H}}, \\
& \langle C(t, u), v\rangle=(\mathcal{C}(t) \varepsilon(u)), \varepsilon(v))_{\mathcal{H}}
\end{aligned}
$$

for $u, v \in V$, a.e. $t \in(0, T)$.

Lemma 8 Under the hypothesis $H(\mathcal{A})$, the operator $A:(0, T) \times V \rightarrow V^{*}$ defined by (9) satisfies $H(A)$ with $a_{0}(t)=\sqrt{2}\left\|\tilde{a_{1}}(t)\right\|_{L^{2}(\Omega)}, a_{1}=\sqrt{2} \tilde{a_{2}}, \alpha=\tilde{a_{3}}$, and $m_{1}=\tilde{a}_{4}$.

Proof By $H(\mathcal{A})($ iii) and Hölder's inequality, we have

$$
\left\|\langle A(t, v), w\rangle \mid \leq \int_{\Omega}\right\| \mathcal{A}(x, t, \varepsilon(v))\left\|_{\mathbb{S}^{d}}\right\| \varepsilon(w)\left\|_{\mathbb{S}^{d}} d x \leq \sqrt{2}\left(\left\|\tilde{a}_{1}(t)\right\|_{L^{2}(\Omega)}+\tilde{a_{2}}\|v\|\right)\right\| w \|
$$

for all $v, w \in V$, a.e. $t \in(0, T)$. Hence the function $(x, t) \mapsto \mathcal{A}(x, t, \varepsilon(v)): \varepsilon(w)$ is integrable for all $v, w \in V$. By Fubini's theorem, we have that $t \mapsto\langle A(t, v), w\rangle$ is measurable for all $v, w \in V$. Hence, for all $v \in V$, the function $t \mapsto A(t, v)$ is weakly measurable from $(0, T)$ into $V^{*}$. Since the latter is separable, from the Pettis measurability theorem, it follows that $t \mapsto A(t, v)$ is measurable for all $v \in V$, i.e. $H(A)$ (i) holds. Next, from $H(\mathcal{A})(\mathrm{iv})$, it follows

$$
\begin{aligned}
\langle A(t, u)-A(t, v), u-v\rangle & =\int_{\Omega}(\mathcal{A}(x, t, \varepsilon(u))-\mathcal{A}(x, t, \varepsilon(v))):(\varepsilon(u)-\varepsilon(v)) d x \\
& \geq \widetilde{a_{4}} \int_{\Omega}\|\varepsilon(u-v)\|_{\mathbb{S}^{d}}^{2} d x=\widetilde{a_{4}}\|u-v\|^{2}
\end{aligned}
$$

for all $u, v \in V$, a.e. $t \in(0, T)$. This shows $H(A)$ (ii). Again from (12) we obtain that $H(A)$ (iii) is satisfied with $a_{0}(t)=\sqrt{2}\left\|\widetilde{a_{1}}(t)\right\|_{L^{2}(\Omega)}$ and $a_{1}=\sqrt{2} \widetilde{a_{2}}$.

From the hypothesis $H(\mathcal{A})(\mathrm{v})$, it follows

$$
\langle A(t, v), v\rangle=\int_{\Omega} \mathcal{A}(x, t, \varepsilon(v)): \varepsilon(v) d x \geq \tilde{a}_{3} \int_{\Omega}\|\varepsilon(v)\|_{\mathbb{S}^{d}}^{2} d x=\tilde{a}_{3}\|v\|^{2}
$$

for all $v \in V$, a.e. $t \in(0, T)$. Hence $H(A)$ (iv) holds with $\alpha=\widetilde{a_{3}}$. Similarly $H(\mathcal{A})($ iv) implies that $A(t, \cdot)$ is monotone for a.e. $t \in(0, T)$. From Proposition 26.12 of Zeidler [44], we know that the operator $A(t, \cdot)$ is continuous for a.e. $t \in(0, T)$. Hence, in particular, it is hemicontinuous proves that $H(A)(v)$ is satisfied and ends the proof of the lemma.

Lemma 9 Under the hypothesis $H(\mathcal{B})$, the operator $B:(0, T) \times V \rightarrow V^{*}$ defined by (10) satisfies $H(B)$ with $L_{B}=L_{\mathcal{B}}, b_{0}(t)=\sqrt{2}\left\|\widetilde{b}_{1}(t)\right\|_{L^{2}(\Omega)}$ and $b_{1}=\sqrt{2} \tilde{b}_{2}$. 
Proof The measurability of $B(\cdot, v)$ for all $v \in V$ is shown analogously as in the proof of Lemma 8. Indeed, using $H(\mathcal{B})$ (ii) and Hölder's inequality, we have

$$
|\langle B(t, v), w\rangle| \leq \sqrt{2}\left(\left\|\widetilde{b_{1}}(t)\right\|_{L^{2}(\Omega)}+\tilde{b_{2}}\|v\|\right)\|w\|
$$

for all $v, w \in V$, a.e. $t \in(0, T)$. From Fubini's theorem, we know that $t \mapsto\langle B(t, v), w\rangle$ is measurable for all $v, w \in V$. Clearly $t \mapsto B(t, v)$ is weakly measurable from $(0, T)$ into $V^{*}$ for all $v \in V$ and since $V^{*}$ is separable, by the Pettis measurability theorem, we deduce that $t \mapsto B(t, v)$ is measurable for all $v \in V$. This proves $H(B)(\mathrm{i})$.

Using (13), we easily obtain that $H(B)$ (iii) holds with $b_{0}(t)=\sqrt{2}\left\|\tilde{b}_{1}(t)\right\|_{L^{2}(\Omega)}$ and $b_{1}=$ $\sqrt{2} \widetilde{b_{2}}$. Next, from $H(\mathcal{B})($ iii) and Hölder's inequality, we get

$$
|\langle B(t, u)-B(t, v), w\rangle| \leq L_{\mathcal{B}} \int_{\Omega}\|\varepsilon(u)-\varepsilon(v)\|_{\mathbb{S}^{d}}\|\varepsilon(w)\|_{\mathbb{S}^{d}} d x \leq L_{\mathcal{B}}\|u-v\|\|w\|
$$

for all $u, v, w \in V$, a.e. $t \in(0, T)$. Hence, $H(B)$ (ii) follows. The proof of the lemma is thus complete.

Lemma 10 Under the hypothesis $H(\mathcal{C})$, the operator $C$ defined by (11) satisfies $H(C)$.

Proof From the hypothesis $H(\mathcal{C})$, we have

$$
\langle C(t, u), v\rangle=\int_{\Omega} c(x, t) \varepsilon(u): \varepsilon(v) d x \quad \text { for } u, v \in V \text {, a.e. } t \in(0, T) .
$$

Since $c(x, t)=\left\{c_{i j k l}(x, t)\right\}$ and $c_{i j k l} \in L^{\infty}(Q)$, using the Hölder inequality we readily obtain that $C \in L^{2}\left(0, T ; \mathcal{L}\left(V, V^{*}\right)\right)$.

We also observe that if $H(f)$ holds then $\left(H_{0}\right)$ is satisfied as well. Now, in order to formulate Problem (HVI) in the form of evolution inclusion, we extend the pointwise relations (6) and (7) to relations involving multifunctions. To this end, we consider the function $g: \Gamma_{C} \times(0, T) \times\left(\mathbb{R}^{d}\right)^{4} \rightarrow \mathbb{R}$ defined by

$$
\begin{aligned}
g(x, t, \zeta, \rho, \xi, \eta)= & j_{1}\left(x, t, \zeta, \rho, \xi_{v}\right)+j_{2}\left(x, t, \zeta, \rho, \eta_{v}\right) \\
& +j_{3}\left(x, t, \zeta, \rho, \xi_{\tau}\right)+j_{4}\left(x, t, \zeta, \rho, \eta_{\tau}\right)
\end{aligned}
$$

for $\zeta, \rho, \xi, \eta \in \mathbb{R}^{d}$ and a.e. $(x, t) \in \Gamma_{C} \times(0, T)$.

In what follows, we will need the following hypotheses.

$\underline{H(g)}$ : The function $g: \Gamma_{C} \times(0, T) \times\left(\mathbb{R}^{d}\right)^{4} \rightarrow \mathbb{R}$ satisfies the following

(i) $g(\cdot, \cdot, \zeta, \rho, \xi, \eta)$ is measurable for all $\zeta, \rho, \xi, \eta \in \mathbb{R}^{d}$, $g(\cdot, \cdot, v(\cdot), w(\cdot), 0,0) \in L^{1}\left(\Gamma_{C} \times(0, T)\right)$ for all $v, w \in L^{2}\left(\Gamma_{C} ; \mathbb{R}^{d}\right) ;$

(ii) $g(x, t, \cdot, \cdot, \xi, \eta)$ is continuous for all $\xi, \eta \in \mathbb{R}^{d}$, a.e. $(x, t) \in \Gamma_{C} \times(0, T)$, $g(x, t, \zeta, \rho, \cdot, \cdot)$ is locally Lipschitz for all $\zeta, \rho \in \mathbb{R}^{d}$, a.e. $(x, t) \in \Gamma_{C} \times(0, T)$;

(iii) $\|\partial g(x, t, \zeta, \rho, \xi, \eta)\|_{\left(\mathbb{R}^{d}\right)^{2}} \leq c_{g 0}+c_{g 1}(\|\zeta\|+\|\xi\|)+c_{g 2}(\|\rho\|+\|\eta\|)$ for all $\zeta, \rho, \xi$, $\eta \in \mathbb{R}^{d}$, a.e. $(x, t) \in \Gamma_{C} \times(0, T)$ with $c_{g 0}, c_{g 1}, c_{g 2} \geq 0$, where $\partial g$ denotes the Clarke subdifferential of $g(x, t, \zeta, \rho, \cdot, \cdot)$; 
(iv) $g^{0}(x, t, \cdot, \cdot, \cdot, \cdot ; \chi, \sigma)$ is upper semicontinuous on $\left(\mathbb{R}^{d}\right)^{4}$ for a.e. $(x, t) \in \Gamma_{C} \times(0, T)$ and all $\chi, \sigma \in \mathbb{R}^{d}$ where $g^{0}$ denotes the generalized directional derivative of Clarke of $g(x, t, \zeta, \rho, \cdot, \cdot)$ in the direction $(\chi, \sigma)$.

$H(g)_{\text {reg }}$ : The function $g: \Gamma_{C} \times(0, T) \times\left(\mathbb{R}^{d}\right)^{4} \rightarrow \mathbb{R}$ is such that either $g(x, t, \zeta, \rho, \cdot, \cdot)$ or $-g(x, t, \zeta, \rho, \cdot, \cdot)$ is regular for all $\zeta, \rho \in \mathbb{R}^{d}$, a.e. $(x, t) \in \Gamma_{C} \times(0, T)$.

Lemma 11 Under the hypotheses $H\left(j_{k}\right)$ for $k=1, \ldots, 4$ and $H(j)_{\text {reg }}$ the function $g$ defined by (14) satisfies $H(g)$ with $c_{g 0}=\max _{1 \leq k \leq 4} c_{k 0}, c_{g 1}=\max \left\{\max _{1 \leq k \leq 4} c_{k 1}, c_{13}, c_{33}\right\}, c_{g 2}=$ $\max \left\{\max _{1 \leq k \leq 4} c_{k 2}, c_{23}, c_{43}\right\}$. Additionally the following equality holds

$$
\begin{aligned}
g^{0}(x, t, \zeta, \rho, \xi, \eta ; \chi, \sigma)= & j_{1}^{0}\left(x, t, \zeta, \rho, \xi_{v} ; \chi_{v}\right)+j_{2}^{0}\left(x, t, \zeta, \rho, \eta_{\nu} ; \sigma_{v}\right) \\
& +j_{3}^{0}\left(x, t, \zeta, \rho, \xi_{\tau} ; \chi_{\tau}\right)+j_{4}^{0}\left(x, t, \zeta, \rho, \eta_{\tau} ; \sigma_{\tau}\right)
\end{aligned}
$$

for $\zeta, \rho, \xi, \eta, \chi, \sigma \in \mathbb{R}^{d}$ and a.e. $(x, t) \in \Gamma_{C} \times(0, T)$ where $j_{k}^{0}$ denotes the generalized directional derivative of $j_{k}(x, t, \zeta, \rho, \cdot)$ for $k=1, \ldots, 4$.

Proof The conditions $H(g)$ (i) and (ii) follow directly from the hypotheses on $j_{k}$ for $k=$ $1, \ldots, 4$. For the proof of (15), let $\zeta, \rho, \xi, \eta \in \mathbb{R}^{d}$ and $(x, t) \in \Gamma_{C} \times(0, T)$. Let us also suppose that $j_{k}(x, t, \zeta, \rho, \cdot)$ for $k=1, \ldots, 4$ are regular in the sense of Clarke. This means, by definition, that for all $r \in \mathbb{R}$ and $\theta \in \mathbb{R}^{d}$ the usual directional derivatives $j_{k}^{\prime}(x, t, \zeta, \rho, r ; s)$ for $k=1,2$ and $j_{k}^{\prime}(x, t, \zeta, \rho, \theta ; \sigma)$ for $k=3,4$ exist and

$$
\begin{cases}j_{k}^{\prime}(x, t, \zeta, \rho, r ; s)=j_{k}^{0}(x, t, \zeta, \rho, r ; s) & \text { for } k=1,2 \\ j_{k}^{\prime}(x, t, \zeta, \rho, \theta ; \sigma)=j_{k}^{0}(x, t, \zeta, \rho, \theta ; \sigma) & \text { for } k=3,4\end{cases}
$$

for all $s \in \mathbb{R}$ and $\sigma \in \mathbb{R}^{d}$. Hence we deduce that the directional derivative $g_{\xi \eta}^{\prime}$ of the function $g(x, t, \zeta, \rho, \cdot, \cdot)$ also exists at every point $(\xi, \eta) \in \mathbb{R}^{d} \times \mathbb{R}^{d}$ and in any direction $(\chi, \sigma) \in$ $\left(\mathbb{R}^{d}\right)^{2}$. By the definition of $g$, we can write $g(x, t, \zeta, \rho, \xi, \eta)=\sum_{k=1}^{4} \widehat{j_{k}}(x, t, \zeta, \rho, \xi, \eta)$, where the functions $\widehat{j_{k}}: \Gamma_{C} \times(0, T) \times\left(\mathbb{R}^{d}\right)^{4} \rightarrow \mathbb{R}$ are defined by

$$
\begin{array}{ll}
\widehat{j_{1}}(x, t, \zeta, \rho, \xi, \eta)=j_{1}\left(x, t, \zeta, \rho, \xi_{v}\right), & \widehat{j_{2}}(x, t, \zeta, \rho, \xi, \eta)=j_{2}\left(x, t, \zeta, \rho, \eta_{v}\right), \\
\widehat{j_{3}}(x, t, \zeta, \rho, \xi, \eta)=j_{3}\left(x, t, \zeta, \rho, \xi_{\tau}\right), & \widehat{j_{4}}(x, t, \zeta, \rho, \xi, \eta)=j_{4}\left(x, t, \zeta, \rho, \eta_{\tau}\right) .
\end{array}
$$

By Proposition 2.3.3 of [6], we have

$$
g^{0}(x, t, \zeta, \rho, \xi, \eta ; \chi, \sigma) \leq \sum_{k=1}^{4}\left(\widehat{j}_{k}\right)^{0}(x, t, \zeta, \rho, \xi, \eta ; \chi, \sigma)
$$

for every direction $(\chi, \sigma) \in\left(\mathbb{R}^{d}\right)^{2}$. Consider now the operators $N_{1} \in \mathcal{L}\left(\mathbb{R}^{d}, \mathbb{R}\right)$ and $N_{2} \in$ $\mathcal{L}\left(\mathbb{R}^{d}, \mathbb{R}^{d}\right)$ given by $N_{1} \xi=\xi_{v}$ and $N_{2} \xi=\xi_{\tau}$ for $\xi \in \mathbb{R}^{d}$. From Theorem 2.3.10 of [6] applied to the functions $j_{k}, k=1, \ldots, k$ and the operators $N_{1}$ and $N_{2}$, we get

$$
\begin{aligned}
& \left(\widehat{j_{1}}\right)^{0}(x, t, \zeta, \rho, \xi, \eta ; \chi, \sigma) \leq j_{1}^{0}\left(x, t, \zeta, \rho, \xi_{v} ; \chi_{v}\right), \\
& \left(\widehat{j_{2}}\right)^{0}(x, t, \zeta, \rho, \xi, \eta ; \chi, \sigma) \leq j_{2}^{0}\left(x, t, \zeta, \rho, \eta_{v} ; \sigma_{v}\right), \\
& \left(\widehat{j_{3}}\right)^{0}(x, t, \zeta, \rho, \xi, \eta ; \chi, \sigma) \leq j_{3}^{0}\left(x, t, \zeta, \rho, \xi_{\tau} ; \chi_{\tau}\right), \\
& \left(\widehat{j_{4}}\right)^{0}(x, t, \zeta, \rho, \xi, \eta ; \chi, \sigma) \leq j_{4}^{0}\left(x, t, \zeta, \rho, \eta_{\tau} ; \sigma_{\tau}\right) .
\end{aligned}
$$


The latter four inequalities together with (17) imply

$$
\begin{aligned}
g^{0}(x, t, \zeta, \rho, \xi, \eta ; \chi, \sigma) \leq & j_{1}^{0}\left(x, t, \zeta, \rho, \xi_{v} ; \chi_{v}\right)+j_{2}^{0}\left(x, t, \zeta, \rho, \eta_{v} ; \sigma_{v}\right) \\
& +j_{3}^{0}\left(x, t, \zeta, \rho, \xi_{\tau} ; \chi_{\tau}\right)+j_{4}^{0}\left(x, t, \zeta, \rho, \eta_{\tau} ; \sigma_{\tau}\right)
\end{aligned}
$$

for $\zeta, \rho, \xi, \eta, \chi, \sigma \in \mathbb{R}^{d}$ and a.e. $(x, t) \in \Gamma_{C} \times(0, T)$. On the other hand, we have

$$
\begin{aligned}
g_{\xi \eta}^{\prime}(x, t, \zeta, \rho, \xi, \eta ; \chi, \sigma) \\
=\lim _{\lambda \downarrow 0} \frac{1}{\lambda}(g(x, t, \zeta, \rho,(\xi, \eta)+\lambda(\chi, \sigma))-g(x, t, \zeta, \rho, \xi, \eta)) \\
=\lim _{\lambda \downarrow 0} \frac{1}{\lambda}\left(j_{1}\left(x, t, \zeta, \rho, \xi_{N}+\lambda \chi_{v}\right)+j_{2}\left(x, t, \zeta, \rho, \eta_{v}+\lambda \sigma_{v}\right)\right. \\
\quad+j_{3}\left(x, t, \zeta, \rho, \xi_{\tau}+\lambda \chi_{\tau}\right)+j_{4}\left(x, t, \zeta, \rho, \eta_{\tau}+\lambda \sigma_{\tau}\right) \\
\left.\quad-j_{1}\left(x, t, \zeta, \rho, \xi_{v}\right)-j_{2}\left(x, t, \zeta, \rho, \eta_{v}\right)-j_{3}\left(x, t, \zeta, \rho, \xi_{\tau}\right)-j_{4}\left(x, t, \zeta, \rho, \eta_{\tau}\right)\right) \\
=j_{1}^{\prime}\left(x, t, \zeta, \rho, \xi_{v} ; \chi_{v}\right)+j_{2}^{\prime}\left(x, t, \zeta, \rho, \eta_{v} ; \sigma_{v}\right)+j_{3}^{\prime}\left(x, t, \zeta, \rho, \xi_{\tau} ; \chi_{\tau}\right) \\
\quad+j_{4}^{\prime}\left(x, t, \zeta, \rho, \eta_{\tau} ; \sigma_{\tau}\right),
\end{aligned}
$$

which together with (18) and (16) implies

$$
g_{\xi \eta}^{\prime}(x, t, \zeta, \rho, \xi, \eta ; \chi, \sigma) \geq g^{0}(x, t, \zeta, \rho, \xi, \eta ; \xi, \sigma)
$$

for all $\xi, \eta, \chi, \sigma \in \mathbb{R}^{d}$. As it follows directly from the definitions the opposite inequality $g_{\xi \eta}^{\prime} \leq g^{0}$ is always true, so we deduce that $g_{\xi \eta}^{\prime}(x, t, \zeta, \rho, \xi, \eta ; \chi, \sigma)=g^{0}(x, t, \zeta, \rho, \xi, \eta$; $\chi, \sigma)$ for $\zeta, \rho, \xi, \eta, \chi, \sigma \in \mathbb{R}^{d}$, a.e. $(x, t) \in \Gamma_{C} \times(0, T)$, which means that $g(x, t, \zeta, \rho, \cdot, \cdot)$ is regular in the sense of Clarke. Thus (15) follows.

If for $k=1, \ldots, k$ the functions $-j_{k}$ are regular in their last variables, then we proceed in the same way as above and deduce the regularity of $-g(x, t, \zeta, \rho, \cdot, \cdot)$ for $(x, t) \in \Gamma_{C} \times(0, T)$ and $\zeta, \rho \in \mathbb{R}^{d}$. We use the property $(-g)^{0}(x, t, \zeta, \rho, \xi, \eta ; \chi, \sigma)=$ $g^{0}(x, t, \zeta, \rho, \xi, \eta ;-\chi,-\sigma)$ for all $\zeta, \rho, \xi, \eta, \chi, \sigma \in \mathbb{R}^{d}$, a.e. $(x, t) \in \Gamma_{C} \times(0, T)$ (cf. Proposition 2.1.1 of [6]), and again deduce (15).

In order to show $H(g)($ iii), let us take $(\bar{\chi}, \bar{\sigma}) \in \partial g(x, t, \zeta, \rho, \xi, \eta)$, where $\zeta, \rho, \xi, \eta, \bar{\chi}$, $\bar{\sigma} \in \mathbb{R}^{d}$ and $(x, t) \in \Gamma_{C} \times(0, T)$. By the definition of the subdifferential and (15), for all $\chi$, $\sigma \in \mathbb{R}^{d}$, we have

$$
\begin{aligned}
\langle(\bar{\chi}, \bar{\sigma}),(\chi, \sigma)\rangle_{\left(\mathbb{R}^{d}\right)^{2}} \leq & g^{0}(x, t, \zeta, \rho, \xi, \eta ; \chi, \sigma) \\
= & j_{1}^{0}\left(x, t, \zeta, \rho, \xi_{v} ; \chi_{\nu}\right)+j_{2}^{0}\left(x, t, \zeta, \rho, \eta_{v} ; \sigma_{\nu}\right)+j_{3}^{0}\left(x, t, \zeta, \rho, \xi_{\tau} ; \chi_{\tau}\right) \\
& \quad+j_{4}^{0}\left(x, t, \zeta, \rho, \eta_{\tau} ; \sigma_{\tau}\right)
\end{aligned}
$$

Using Proposition 2.1.2 of [6] and $H\left(j_{1}\right)$ (iii), we deduce

$$
j_{1}^{0}\left(x, t, \zeta, \rho, \xi_{v} ; \chi_{v}\right) \leq\left|\chi_{v}\right|\left(c_{10}+c_{11}\|\zeta\|+c_{12}\|\rho\|+c_{13}\left|\xi_{v}\right|\right)
$$

Analogously, by $H\left(j_{k}\right)_{1}$ (iii) for $k=2,3$, 4, we obtain the similar inequalities. Recalling that $\left|\xi_{v}\right| \leq\|\xi\|$ and $\left\|\xi_{\tau}\right\| \leq\|\xi\|$ for all $\xi \in \mathbb{R}^{d}$, from the above, we have

$$
\left\langle(\bar{\chi}, \bar{\sigma}),\left.(\chi, \sigma)\right|_{\left(\mathbb{R}^{d}\right)^{2}} \leq\left(c_{g 0}+c_{g 1}(\|\zeta\|+\|\xi\|)+c_{g 2}(\|\rho\|+\|\eta\|)\right)\|(\chi, \sigma)\|_{\left(\mathbb{R}^{d}\right)^{2}},\right.
$$


where $c_{g 0}=\max c_{k 0}, c_{g 1}=\max \left\{\max c_{k 1}, c_{13}, c_{33}\right\}, c_{g 2}=\max \left\{\max c_{k 2}, c_{23}, c_{43}\right\}$. Hence $H(g)$ (iii) holds.

Finally, we suppose the hypotheses $H\left(j_{k}\right)(i v)$ for $k=1, \ldots, 4$ and $H(j)_{\text {reg }}$. In order to prove $H(g)(i v)$, it is enough to show that $g^{0}(x, t, \cdot, \cdot, \cdot, \cdot ; \chi, \sigma)$ is upper semicontinuous on $\left(\mathbb{R}^{d}\right)^{4}$ for all $\chi, \sigma \in \mathbb{R}^{d}$ and a.e. $(x, t) \in \Gamma_{C} \times(0, T)$. Let $\chi, \sigma \in \mathbb{R}^{d}$ and $(x, t) \in \Gamma_{C} \times(0, T)$, and let $\left\{\zeta_{n}\right\},\left\{\rho_{n}\right\},\left\{\xi_{n}\right\},\left\{\eta_{n}\right\}$ be sequences in $\mathbb{R}^{d}$ such that $\zeta_{n} \rightarrow \zeta, \rho_{n} \rightarrow \rho, \xi_{n} \rightarrow \xi$ and $\eta_{n} \rightarrow \eta$. By the hypothesis $H\left(j_{k}\right)(i v)$ for $k=1, \ldots, 4$ and equality (15), we find

$$
\begin{aligned}
& \lim \sup g^{0}\left(x, t, \zeta_{n}, \rho_{n}, \xi_{n}, \eta_{n} ; \chi, \sigma\right) \\
& \leq \limsup j_{1}^{0}\left(x, t, \zeta_{n}, \rho_{n}, \xi_{n v} ; \chi_{v}\right)+\limsup j_{2}^{0}\left(x, t, \zeta_{n}, \rho_{n}, \eta_{n v} ; \sigma_{v}\right) \\
& +\limsup j_{3}^{0}\left(x, t, \zeta_{n}, \rho_{n}, \xi_{n \tau} ; \chi_{\tau}\right)+\limsup j_{4}^{0}\left(x, t, \zeta_{n}, \rho_{n}, \eta_{n \tau} ; \sigma_{\tau}\right) \\
& \leq j_{1}^{0}\left(x, t, \zeta, \rho, \xi_{v} ; \chi_{v}\right)+j_{2}^{0}\left(x, t, \zeta, \rho, \eta_{v} ; \sigma_{v}\right) \\
& +j_{3}^{0}\left(x, t, \zeta, \rho, \xi_{\tau} ; \chi_{\tau}\right)+j_{4}^{0}\left(x, t, \zeta, \rho, \eta_{\tau} ; \sigma_{\tau}\right)=g^{0}(x, t, \zeta, \rho, \xi, \eta ; \chi, \sigma) .
\end{aligned}
$$

Hence the condition $H(g)(i v)$ follows. The proof of the lemma is complete.

The next step is to study the integral functional corresponding to superpotentials which appear in the boundary conditions. Let us consider the functional $G:(0, T) \times$ $L^{2}\left(\Gamma_{C} ; \mathbb{R}^{d}\right)^{4} \rightarrow \mathbb{R}$ defined by

$$
G(t, w, z, u, v)=\int_{\Gamma_{C}} g(x, t, w(x), z(x), u(x), v(x)) d \Gamma
$$

for $w, z, u, v \in L^{2}\left(\Gamma_{C} ; \mathbb{R}^{d}\right), t \in(0, T)$, where the integrand $g$ is given by (14).

We introduce the following hypothesis.

$H(G):$ The functional $G:(0, T) \times L^{2}\left(\Gamma_{C} ; \mathbb{R}^{d}\right)^{4} \rightarrow \mathbb{R}$ is such that

(i) $G(\cdot, w, z, u, v)$ is measurable for all $w, z, u, v \in L^{2}\left(\Gamma_{C} ; \mathbb{R}^{d}\right)$,

$G(\cdot, w, z, 0,0) \in L^{1}(0, T)$ for all $w, z \in L^{2}\left(\Gamma_{C} ; \mathbb{R}^{d}\right)$

(ii) $G(t, w, z, \cdot, \cdot)$ is Lipschitz continuous on bounded subsets of $L^{2}\left(\Gamma_{C} ; \mathbb{R}^{d}\right)^{2}$ for all $w$, $z \in L^{2}\left(\Gamma_{C} ; \mathbb{R}^{d}\right)$, a.e. $t \in(0, T)$

(iii) $\|\partial G(t, w, z, u, v)\|_{L^{2}\left(\Gamma_{C} ; \mathbb{R}^{d}\right)^{2}} \leq c_{G 0}+c_{G 1}(\|w\|+\|u\|)+c_{G 2}(\|z\|+\|v\|)$ for all $w$, $z, u, v \in L^{2}\left(\Gamma_{C} ; \mathbb{R}^{d}\right)$, a.e. $t \in(0, T)$ with $c_{G 0}, c_{G 1}, c_{G 2} \geq 0$, where $\partial G$ denotes the Clarke subdifferential of $G(t, w, z, \cdot, \cdot)$;

(iv) For all $w, z, u, v, \bar{u}, \bar{v} \in L^{2}\left(\Gamma_{C} ; \mathbb{R}^{d}\right)$, a.e. $t \in(0, T)$, we have

$$
G^{0}(t, w, z, u, v ; \bar{u}, \bar{v})=\int_{\Gamma_{C}} g^{0}(x, t, w(x), z(x), u(x), v(x) ; \bar{u}(x), \bar{v}(x)) d \Gamma,
$$

where $G^{0}$ denotes the generalized directional derivative of $G(t, w, z, \cdot, \cdot)$ at a point $(u, v)$ in the direction $(\bar{u}, \bar{v})$;

(v) $G^{0}(t, \cdot, \cdot, \cdot, \cdot ; \bar{u}, \bar{v})$ is upper semicontinuous on $L^{2}\left(\Gamma_{C} ; \mathbb{R}^{d}\right)^{4}$ for all $\bar{u}, \bar{v} \in L^{2}\left(\Gamma_{C} ; \mathbb{R}^{d}\right)$, a.e. $t \in(0, T)$.

$H(G)_{\text {reg }}:$ The functional $G:(0, T) \times L^{2}\left(\Gamma_{C} ; \mathbb{R}^{d}\right)^{4} \rightarrow \mathbb{R}$ is such that either $G(t, w, z, \cdot, \cdot)$

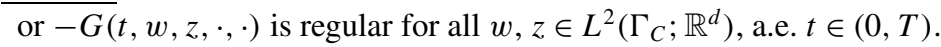

Lemma 12 Under the hypotheses $H(g)$ and $H(g)_{\text {reg }}$ hold the functional $G$ defined by (19) satisfies $H(G)$ with $c_{G 0}=c_{g 0} \sqrt{5 m\left(\Gamma_{C}\right)}, c_{G 1}=c_{g 1} \sqrt{5}, c_{G 2}=c_{g 2} \sqrt{5}$ and $H(G)_{\text {reg }}$. 
Proof First, from $H(g)$ (ii) and Lemma 1, it follows that $g(x, t, \cdot, \cdot, \cdot, \cdot)$ is continuous on $\left(\mathbb{R}^{d}\right)^{4}$ which together with $H(g)(\mathrm{i})$ implies that $g$ is a Carathéodory function. Hence $(x, t) \mapsto g(x, t, w(x), z(x), u(x), v(x))$ is measurable for all $w, z, u, v \in L^{2}\left(\Gamma_{C} ; \mathbb{R}^{d}\right)$ and subsequently the integrand of (19) is a measurable function of $x$.

Next, applying the Lebourg mean value theorem (cf., e.g., Theorem 5.6 .25 of [7]) to a locally Lipschitz function $g(x, t, \zeta, \rho, \cdot, \cdot)$ (cf. $H(g)($ ii) $)$, we deduce that there exist $(\bar{\xi}, \bar{\eta})$ in the interval $[0,(\xi, \eta)] \subset\left(\mathbb{R}^{d}\right)^{2}$ and $\left(\xi^{*}, \eta^{*}\right) \in \partial g(x, t, \zeta, \rho, \bar{\xi}, \bar{\eta})$ such that

$$
g(x, t, \zeta, \rho, \xi, \eta)-g(x, t, \zeta, \rho, 0,0)=\left(\left(\xi^{*}, \eta^{*}\right),(\xi, \eta)\right)_{\left(\mathbb{R}^{d}\right)^{2}}
$$

for all $\zeta, \rho, \xi, \eta \in \mathbb{R}^{d}$, a.e. $(x, t) \in \Gamma_{C} \times(0, T)$. Hence, by $H(g)$ (iii), we obtain

$$
\begin{aligned}
& g(x, t, w(x), z(x), u(x), v(x)) \\
& \quad \leq g(x, t, w(x), z(x), 0,0) \\
& \quad+c(\|u(x)\|+\|v(x)\|)\left(c_{g 0}+c_{g 1}(\|w(x)\|+\|u(x)\|)+c_{g 2}(\|z(x)\|+\|v(x)\|)\right)
\end{aligned}
$$

for all $w, z, u, v \in L^{2}\left(\Gamma_{C} ; \mathbb{R}^{d}\right)$, a.e. $(x, t) \in \Gamma_{C} \times(0, T)$ with a constant $c>0$. From $H(g)(\mathrm{i})$, it is easy to see that $(x, t) \mapsto g(x, t, w(x), z(x), u(x), v(x))$ is integrable and from Fubini's theorem, we infer that $G(\cdot, w, z, u, v)$ is measurable and $H(G)$ (i) holds.

Now, let $w, z \in L^{2}\left(\Gamma_{C} ; \mathbb{R}^{d}\right)$ and let $\tilde{g}: \Gamma_{C} \times(0, T) \times\left(\mathbb{R}^{d}\right)^{2} \rightarrow \mathbb{R}$ be defined by

$$
\widetilde{g}(x, t, \xi, \eta)=g(x, t, w(x), z(x), \xi, \eta) \quad \text { for } \xi, \eta \in \mathbb{R}^{d} \text {, a.e. }(x, t) \in \Gamma_{C} \times(0, T) \text {. }
$$

From (i) and (ii) of $H(g)$, it follows that $\tilde{g}(\cdot, \cdot, \xi, \eta)$ is measurable for all $\xi, \eta \in \mathbb{R}^{d}$, $\tilde{g}(\cdot, t, 0,0) \in L^{1}\left(\Gamma_{C}\right)$ for a.e. $t \in(0, T)$ (by invoking again Fubini's theorem) and $\widetilde{g}(x, t, \cdot, \cdot)$ is locally Lipschitz for a.e. $(x, t) \in \Gamma_{C} \times(0, T)$. Moreover, by employing $H(g)$ (iii), we have

$$
\begin{aligned}
\|\partial \widetilde{g}(x, t, \xi, \eta)\|_{\left(\mathbb{R}^{d}\right)^{2}} & =\|\partial g(x, t, w(x), z(x), \xi, \eta)\|_{\left(\mathbb{R}^{d}\right)^{2}} \\
& \leq c_{g 0}+c_{g 1}(\|w(x)\|+\|\xi\|)+c_{g 2}(\|z(x)\|+\|\eta\|) \\
& =\omega(x)+\max \left\{c_{g 1}, c_{g 2}\right\}(\|\xi\|+\|\eta\|)
\end{aligned}
$$

with $\omega \in L^{2}\left(\Gamma_{C}\right)$. At this stage we use Aubin-Clarke's theorem (cf. Theorem 5.6.39 of [7]) to deduce that the functional $G(t, w, z, \cdot, \cdot)$ is well defined, finite and Lipschitz continuous on bounded subsets of $L^{2}\left(\Gamma_{C} ; \mathbb{R}^{d}\right)$ for all $w, z \in L^{2}\left(\Gamma_{C} ; \mathbb{R}^{d}\right)$, a.e. $t \in(0, T)$. Hence $H(G)$ (ii) is satisfied. Furthermore, for $w, z, u, v \in L^{2}\left(\Gamma_{C} ; \mathbb{R}^{d}\right)$ and a.e. $t \in(0, T)$, we have

$$
\begin{aligned}
& \partial G(t, w, z, u, v) \\
& \quad \subset\left\{(\bar{u}, \bar{v}) \in L^{2}\left(\Gamma_{C} ; \mathbb{R}^{d}\right)^{2} \mid(\bar{u}(x), \bar{v}(x)) \in \partial g(x, t, w(x), z(x), u(x), v(x)) \text { a.e. } x \in \Gamma_{C}\right\} .
\end{aligned}
$$

Hence, by $H(g)$ (iii), we thus obtain that for all $(\bar{u}, \bar{v}) \in \partial G(t, w, z, u, v), \bar{u}, \bar{v} \in L^{2}\left(\Gamma_{C} ; \mathbb{R}^{d}\right)$, we have

$$
\|(\bar{u}, \bar{v})\|_{L^{2}\left(\Gamma_{C} ; \mathbb{R}^{d}\right)^{2}} \leq \sqrt{5}\left(c_{g 0} \sqrt{m\left(\Gamma_{C}\right)}+c_{g 1}(\|w\|+\|u\|)+c_{g 2}(\|z\|+\|v\|)\right),
$$

which entails that the condition $H(G)$ (iii) holds with the aforementioned constants $c_{g 0}, c_{g 1}$ and $c_{g 2}$. 
Next, by the Fatou lemma, we have

$$
\begin{aligned}
& G^{0}(t, w, z, u, v ; \bar{u}, \bar{v}) \\
& \leq \int_{\Gamma_{C}\left(u^{\prime}, v^{\prime}\right) \rightarrow(u, v), \lambda \downarrow 0} \lim _{\lambda} \frac{1}{\lambda}\left(g\left(x, t, w(x), z(x), u^{\prime}(x)+\lambda \bar{u}(x), v^{\prime}(x)+\lambda \bar{v}(x)\right)\right. \\
& \left.\quad-g\left(x, t, w(x), z(x), u^{\prime}(x), v^{\prime}(x)\right)\right) d \Gamma \\
& \quad=\int_{\Gamma_{C}} g^{0}(x, t, w(x), z(x), u(x), v(x) ; \bar{u}(x), \bar{v}(x)) d \Gamma
\end{aligned}
$$

for all $w, z, u, v, \bar{u}, \bar{v} \in L^{2}\left(\Gamma_{C} ; \mathbb{R}^{d}\right)$, a.e. $t \in(0, T)$.

Since the function $g(x, t, \zeta, \rho, \cdot, \cdot)$ is regular in the sense of Clarke. By exploiting the Fatou lemma and the above, we obtain

$$
\begin{aligned}
& G^{0}(t, w, z, u, v ; \bar{u}, \bar{v}) \\
& \geq \underset{\lambda \downarrow 0}{\liminf } \frac{1}{\lambda}(G(t, w, z,(u, v)+\lambda(\bar{u}, \bar{v}))-G(t, w, z, u, v)) \\
& \geq \int_{\Gamma_{C}} \liminf _{\lambda \downarrow 0} \frac{1}{\lambda}(g(x, t, w(x), z(x), u(x)+\lambda \bar{u}(x), v(x)+\lambda \bar{v}(x)) \\
& \quad-g(x, t, w(x), z(x), u(x), v(x))) d \Gamma \\
& =\int_{\Gamma_{C}} g^{0}(x, t, w(x), z(x), u(x), v(x) ; \bar{u}(x), \bar{v}(x)) d \Gamma \geq G^{0}(t, w, z, u, v ; \bar{u}, \bar{v})
\end{aligned}
$$

for all $w, z, u, v, \bar{u}, \bar{v} \in L^{2}\left(\Gamma_{C} ; \mathbb{R}^{d}\right)$, a.e. $t \in(0, T)$. Hence $G_{(u, v)}^{\prime}(t, w, z, u, v ; \bar{u}, \bar{v})$ exists and

$$
G_{(u, v)}^{\prime}(t, w, z, u, v ; \bar{u}, \bar{v})=G^{0}(t, w, z, u, v ; \bar{u}, \bar{v})
$$

which means that $G(t, w, z, \cdot, \cdot)$ is regular for all $w, z \in L^{2}\left(\Gamma_{C} ; \mathbb{R}^{d}\right)$ and a.e. $t \in(0, T)$. The two above also imply that $H(G)(i v)$ holds.

When $-g(x, t, \zeta, \rho, \cdot, \cdot)$ is regular in the sense of Clarke, we proceed analogously as above and deduce the regularity of $-G(t, w, z, \cdot, \cdot)$. From the property

$$
(-G)^{0}(t, w, z, u, v ; \bar{u}, \bar{v})=G^{0}(t, w, z, u, v ;-\bar{u},-\bar{v})
$$

for all $w, z, u, v, \bar{u}, \bar{v} \in L^{2}\left(\Gamma_{C} ; \mathbb{R}^{d}\right)$, a.e. $t \in(0, T)$ (cf. Proposition 2.1.1 of [6]), we again get equality (20).

Finally, we suppose the hypotheses $H(g)(i v)$. Let $t \in(0, T), w, z, u, v, \bar{u}, \bar{v} \in$ $L^{2}\left(\Gamma_{C} ; \mathbb{R}^{d}\right)$ and $\left\{w_{n}\right\},\left\{z_{n}\right\},\left\{u_{n}\right\},\left\{v_{n}\right\}$ be sequences in $L^{2}\left(\Gamma_{C} ; \mathbb{R}^{d}\right)$ such that $w_{n} \rightarrow w$, $z_{n} \rightarrow z, u_{n} \rightarrow u$ and $v_{n} \rightarrow v$ in $L^{2}\left(\Gamma_{C} ; \mathbb{R}^{d}\right)$. We may assume by passing to subsequences, if necessary, that $w_{n}(x) \rightarrow w(x), z_{n}(x) \rightarrow z(x), u_{n}(x) \rightarrow u(x)$ and $v_{n}(x) \rightarrow v(x)$ in $\mathbb{R}^{d}$ for a.e. $x \in \Gamma_{C},\left\|w_{n}(x)\right\| \leq w_{0}(x),\left\|z_{n}(x)\right\| \leq z_{0}(x),\left\|u_{n}(x)\right\| \leq u_{0}(x)$ and $\left\|v_{n}(x)\right\| \leq v_{0}(x)$ with $w_{0}, z_{0}, u_{0}, v_{0} \in L^{2}\left(\Gamma_{C} ; \mathbb{R}^{d}\right)$. By the Fatou lemma and $H(g)(i v)$, we obtain

$$
\begin{aligned}
& \lim \sup G^{0}\left(t, w_{n}, z_{n}, u_{n}, v_{n}, \bar{u}, \bar{v}\right) \\
& \quad=\limsup \int_{\Gamma_{C}} g^{0}\left(x, t, w_{n}(x), z_{n}(x), u_{n}(x), v_{n}(x) ; \bar{u}(x), \bar{v}(x)\right) d \Gamma
\end{aligned}
$$




$$
\begin{aligned}
& \leq \int_{\Gamma_{C}} \lim \sup g^{0}\left(x, t, w_{n}(x), z_{n}(x), u_{n}(x), v_{n}(x) ; \bar{u}(x), \bar{v}(x)\right) d \Gamma \\
& \leq \int_{\Gamma_{C}} g^{0}(x, t, w(x), z(x), u(x), v(x) ; \bar{u}(x), \bar{v}(x)) d \Gamma=G^{0}(t, w, z, u, v, \bar{u}, \bar{v})
\end{aligned}
$$

for all $\bar{u}, \bar{v} \in L^{2}\left(\Gamma_{C} ; \mathbb{R}^{d}\right)$ and a.e. $t \in(0, T)$. This means that $G^{0}(t, \cdot, \cdot, \cdot, \cdot, \bar{u}, \bar{v})$ is upper semicontinuous on $L^{2}\left(\Gamma_{C} ; \mathbb{R}^{d}\right)^{4}$ for all $\bar{u}, \bar{v} \in L^{2}\left(\Gamma_{C} ; \mathbb{R}^{d}\right)$ and a.e. $t \in(0, T)$. This completes the proof that the functional $G$ satisfies $H(G)(v)$. The proof of the lemma is done.

Now we are in a position to carry out the last step of the construction of the multifunction which will appear in the evolution inclusion. We introduce the following operators

$$
\begin{array}{lll}
R: Z \times Z \rightarrow L^{2}\left(\Gamma_{C} ; \mathbb{R}^{d}\right)^{2} & \text { by } R\left(z_{1}, z_{2}\right)=\left(\gamma z_{1}, \gamma z_{2}\right) & \text { for all } z_{1}, z_{2} \in Z, \\
R^{*}: L^{2}\left(\Gamma_{C} ; \mathbb{R}^{d}\right)^{2} \rightarrow Z^{*} \times Z^{*} & \text { by } R^{*}(u, v)=\left(\gamma^{*} u, \gamma^{*} v\right) & \text { for all } u, v \in L^{2}\left(\Gamma_{C} ; \mathbb{R}^{d}\right), \\
S: Z^{*} \times Z^{*} \rightarrow Z^{*} & \text { by } S\left(z_{1}^{*}, z_{2}^{*}\right)=z_{1}^{*}+z_{2}^{*} & \text { for all } z_{1}^{*}, z_{2}^{*} \in Z^{*} .
\end{array}
$$

We define the following multivalued mapping $F:(0, T) \times V \times V \rightarrow 2^{Z^{*}}$ by

$$
F(t, u, v)=S R^{*} \partial G(t, R(u, v), R(u, v)) \quad \text { for } u, v \in V \text {, a.e. } t \in(0, T),
$$

where $\partial G$ denotes the Clarke subdifferential of the functional $G=G(t, w, z, u, v)$ defined by (19) with respect to $(u, v)$.

Before we establish the properties of the multifunction $F$ given by (22), we need the following auxiliary lemma (cf. [18]).

Lemma 13 Let $(\Omega, \Sigma)$ be a measurable space, $Y_{1}, Y_{2}$ be separable Banach spaces, $A \in \mathcal{L}\left(Y_{1}, Y_{2}\right)$ and let $G: \Omega \rightarrow \mathcal{P}_{w k c}\left(Y_{1}\right)$ be measurable. Then the multifunction $F: \Omega \rightarrow$ $\mathcal{P}_{w k c}\left(Y_{2}\right)$ given by $F(\omega)=A G(\omega)$ for $\omega \in \Omega$ is measurable.

Lemma 14 Under $H(G)$ and $H(G)_{\text {reg }}$, the multifunction $F:(0, T) \times V \times V \rightarrow 2^{Z^{*}}$ defined by (22) satisfies $H(F)$ with $d_{0}(t)=c_{G 0}\|\gamma\|, d_{1}=2 c_{e} c_{G 1}\|\gamma\|^{2}$ and $d_{2}=2 c_{e} c_{G 2}\|\gamma\|^{2}$.

Proof The fact that the mapping $F$ has nonempty and convex values follows from the nonemptiness and convexity of values of the Clarke subdifferential of $G$ (cf. Proposition 2.1.2 of [6]). Because the values of the subdifferential $\partial G(t, w, z, \cdot, \cdot)$ are weakly closed subsets of $L^{2}\left(\Gamma_{C} ; \mathbb{R}^{d}\right)$, using $H(G)_{1}$, we can also easily check that the mapping $F$ has closed values in $Z^{*}$.

To show that $F(\cdot, u, v)$ is measurable on $(0, T)$ for all $u, v \in V$, let $w, z, \bar{u}$, $\bar{v} \in L^{2}\left(\Gamma_{C} ; \mathbb{R}^{d}\right)$. Since, by the hypothesis $H(G)_{1}, G(\cdot, w, z, \bar{u}, \bar{v})$ is measurable and $G(t, w, z, \cdot, \cdot)$ is locally Lipschitz on $L^{2}\left(\Gamma_{C} ; \mathbb{R}^{d}\right)^{2}$ (being Lipschitz continuous on bounded subsets) for a.e. $t \in(0, T)$, according to Lemma 2 , we know that

$$
(0, T) \times L^{2}\left(\Gamma_{C} ; \mathbb{R}^{d}\right)^{2} \ni(t, \bar{u}, \bar{v}) \quad \mapsto \quad \partial G(t, w, z, \bar{u}, \bar{v}) \subset L^{2}\left(\Gamma_{C} ; \mathbb{R}^{d}\right)^{2}
$$

is measurable. Hence, by Proposition 2.4.3 of [7], we infer that also the multifunction $(0, T) \ni t \mapsto \partial G(t, w, z, \bar{u}, \bar{v})$ is measurable, and clearly it is $\mathcal{P}_{w k c}\left(L^{2}\left(\Gamma_{C} ; \mathbb{R}^{d}\right)^{2}\right)$-valued. On the other hand, we can readily verify that $S R^{*}: L^{2}\left(\Gamma_{C} ; \mathbb{R}^{d}\right)^{2} \rightarrow Z^{*}$ is a linear continuous operator. These properties ensure the applicability of Lemma 13. So we have 
that $(0, T) \ni t \mapsto S R^{*} \partial G(t, w, z, \bar{u}, \bar{v})$ is measurable. As a consequence the multifunction $F(\cdot, u, v)$ is measurable for all $u, v \in V$.

Next we will prove the upper semicontinuity of $F(t, \cdot, \cdot)$ for a.e. $t \in(0, T)$. According to Proposition 4.1.4 of [7], we show that for every weakly closed subset $K$ of $Z^{*}$, the set

$$
F^{-}(K)=\{(u, v) \in V \times V \mid F(t, u, v) \cap K \neq \emptyset\}
$$

is closed in $Z \times Z$. Let $t \in(0, T),\left\{\left(u_{n}, v_{n}\right)\right\} \subset F^{-}(K)$ and $\left(u_{n}, v_{n}\right) \rightarrow(u, v)$ in $Z \times Z$. We can find $\zeta_{n} \in F\left(t, u_{n}, v_{n}\right) \cap K$ for $n \in \mathbb{N}$. By the definition of $F$, we have $\zeta_{n}=\zeta_{n}^{1}+\zeta_{n}^{2}$, $\left(\zeta_{n}^{1}, \zeta_{n}^{2}\right)=\left(\gamma^{*} \eta_{n}^{1}, \gamma^{*} \eta_{n}^{2}\right)$ with $\left(\eta_{n}^{1}, \eta_{n}^{2}\right) \in L^{2}\left(\Gamma_{C} ; \mathbb{R}^{d}\right)$ and

$$
\left(\eta_{n}^{1}, \eta_{n}^{2}\right) \in \partial G\left(t, \gamma u_{n}, \gamma v_{n}, \gamma u_{n}, \gamma v_{n}\right) \quad \text { for a.e. } t \in(0, T) .
$$

Using the continuity of the trace operator, we have $\gamma u_{n} \rightarrow \gamma u, \gamma v_{n} \rightarrow \gamma v$ in $L^{2}\left(\Gamma_{C} ; \mathbb{R}^{d}\right)$. Since by $H(G)$ (iii) the operator $\partial G(t, \cdot, \cdot, \cdot, \cdot)$ is bounded (it maps bounded sets into bounded sets), from (23), it follows that the sequence $\left\{\left(\eta_{n}^{1}, \eta_{n}^{2}\right)\right\}$ remains in a bounded subset of $L^{2}\left(\Gamma_{C} ; \mathbb{R}^{d}\right)^{2}$. Thus, by passing to a subsequence, if necessary, we may suppose that

$$
\eta_{n}^{1} \rightarrow \eta^{1}, \quad \eta_{n}^{2} \rightarrow \eta^{2} \quad \text { weakly in } L^{2}\left(\Gamma_{C} ; \mathbb{R}^{d}\right)
$$

for some $\eta^{1}, \eta^{2} \in L^{2}\left(\Gamma_{C} ; \mathbb{R}^{d}\right)$. Now, we will use the fact that the graph of $\partial G(t, \cdot, \cdot, \cdot, \cdot)$ is closed in $L^{2}\left(\Gamma_{C} ; \mathbb{R}^{d}\right)^{4} \times\left(w-L^{2}\left(\Gamma_{C} ; \mathbb{R}^{d}\right)^{2}\right)$-topology for a.e. $t \in(0, T)$, which will be showed at the end of this proof. Hence and from (23), we obtain

$$
\left(\eta^{1}, \eta^{2}\right) \in \partial G(t, \gamma u, \gamma v, \gamma u, \gamma v)
$$

Furthermore, since $\left\{\zeta_{n}\right\}$ also remains in a bounded subset of $Z^{*}$, we may assume that $\zeta_{n} \rightarrow \zeta$ weakly in $Z^{*}$. Because $\zeta_{n} \in K$ and $K$ is weakly closed in $Z^{*}$, it follows that $\zeta \in K$. By the continuity and linearity of the operator $\gamma^{*}$, we obtain

$$
\gamma^{*} \eta_{n}^{1} \rightarrow \gamma^{*} \eta^{1}, \quad \gamma^{*} \eta_{n}^{2} \rightarrow \gamma^{*} \eta^{2} \quad \text { weakly in } Z^{*} .
$$

Hence

$$
\zeta_{n}=\gamma^{*} \eta_{n}^{1}+\gamma^{*} \eta_{n}^{2} \rightarrow \gamma^{*} \eta^{1}+\gamma^{*} \eta^{2}=\zeta^{1}+\zeta^{2} \text { weakly in } Z^{*}
$$

and $\zeta=\zeta^{1}+\zeta^{2}$, where $\left(\zeta^{1}, \zeta^{2}\right)=\left(\gamma^{*} \eta^{1}, \gamma^{*} \eta^{2}\right)$ and $\left(\eta^{1}, \eta^{2}\right) \in \partial G(t, \gamma u, \gamma v, \gamma u, \gamma v)$. This, by the definition of $F$ implies that $\zeta \in F(t, u, v)$. As a consequence, once $\zeta \in K$, we know that $F^{-}(K)$ is closed in $Z \times Z$. Hence $H(F)$ (ii) follows.

Next, we show that $F$ satisfies $H(F)$ (iii). Let $t \in(0, T), u, v \in V$ and $z^{*} \in Z^{*}, z^{*} \in$ $F(t, u, v)$. The latter is equivalent to $z^{*}=z_{1}^{*}+z_{2}^{*}, z_{1}^{*}, z_{2}^{*} \in Z^{*},\left(z_{1}^{*}, z_{2}^{*}\right)=\left(\gamma^{*} \eta_{1}, \gamma^{*} \eta_{2}\right)$ where $\eta_{1}, \eta_{2} \in L^{2}\left(\Gamma_{C} ; \mathbb{R}^{d}\right)$ and $\left(\eta_{1}, \eta_{2}\right) \in \partial G(t, \gamma u, \gamma v, \gamma u, \gamma v)$. Using the estimate $H(G)(\mathrm{iii})$, we have

$$
\begin{aligned}
\left\|z^{*}\right\|_{Z^{*}} & \leq\left\|\gamma^{*}\right\|\left\|\eta_{1}+\eta_{2}\right\|_{L^{2}\left(\Gamma_{C} ; \mathbb{R}^{d}\right)} \leq\left\|\gamma^{*}\right\|\left(c_{G 0}+2 c_{G 1}\|\gamma u\|_{L^{2}\left(\Gamma_{C} ; \mathbb{R}^{d}\right)}+2 c_{G 2}\|\gamma v\|_{L^{2}\left(\Gamma_{C} ; \mathbb{R}^{d}\right)}\right) \\
& \leq c_{G 0}\|\gamma\|+2 c_{e} c_{G 1}\|\gamma\|^{2}\|u\|+2 c_{e} c_{G 2}\|\gamma\|^{2}\|v\|
\end{aligned}
$$

where $\left\|\gamma^{*}\right\|=\|\gamma\|$ denotes the norm in $\mathcal{L}\left(L^{2}\left(\Gamma_{C} ; \mathbb{R}^{d}\right), Z^{*}\right)$ and $c_{e}>0$ is the embedding constant of $V$ into $Z$. This implies that $F$ satisfies $H(F)\left(\right.$ iii) with $d_{0}(t)=c_{G 0}\|\gamma\|, d_{1}=$ $2 c_{e} c_{G 1}\|\gamma\|^{2}$ and $d_{2}=2 c_{e} c_{G 2}\|\gamma\|^{2}$. 
To complete the proof, it is enough to show that the graph of $\partial G(t, \cdot, \cdot, \cdot, \cdot)$ is closed in $L^{2}\left(\Gamma_{C} ; \mathbb{R}^{d}\right)^{4} \times\left(w-L^{2}\left(\Gamma_{C} ; \mathbb{R}^{d}\right)^{2}\right)$-topology for a.e. $t \in(0, T)$. This is a simple consequence of $H(G)(v)$. Indeed, let $t \in(0, T),\left\{w_{n}\right\},\left\{z_{n}\right\},\left\{u_{n}\right\},\left\{v_{n}\right\}$ be sequences in $L^{2}\left(\Gamma_{C} ; \mathbb{R}^{d}\right)$ such that $w_{n} \rightarrow w, z_{n} \rightarrow z, u_{n} \rightarrow u, v_{n} \rightarrow v$ in $L^{2}\left(\Gamma_{C} ; \mathbb{R}^{d}\right)$, let $\left\{\left(\eta_{n}^{1}, \eta_{n}^{2}\right)\right\} \subset L^{2}\left(\Gamma_{C} ; \mathbb{R}^{d}\right)^{2}$, $\left(\eta_{n}^{1}, \eta_{n}^{2}\right) \rightarrow\left(\eta^{1}, \eta^{2}\right)$ weakly in $L^{2}\left(\Gamma_{C} ; \mathbb{R}^{d}\right)^{2}$ and $\left(\eta_{n}^{1}, \eta_{n}^{2}\right) \in \partial G\left(t, w_{n}, z_{n}, u_{n}, v_{n}\right)$. The latter means that

$$
\left\langle\left(\eta_{n}^{1}, \eta_{n}^{2}\right),(\bar{u}, \bar{v})\right\rangle_{L^{2}\left(\Gamma_{C} ; \mathbb{R}^{d}\right)^{2}} \leq G^{0}\left(t, w_{n}, z_{n}, u_{n}, v_{n} ; \bar{u}, \bar{v}\right) \quad \text { for all } \bar{u}, \bar{v} \in L^{2}\left(\Gamma_{C} ; \mathbb{R}^{d}\right)
$$

The hypothesis $H(G)(v)$ implies

$$
\left\langle\left(\eta^{1}, \eta^{2}\right),\left.(\bar{u}, \bar{v})\right|_{L^{2}\left(\Gamma_{C} ; \mathbb{R}^{d}\right)^{2}} \leq \lim \sup G^{0}\left(t, w_{n}, z_{n}, u_{n}, v_{n} ; \bar{u}, \bar{v}\right) \leq G^{0}(t, w, z, u, v ; \bar{u}, \bar{v})\right.
$$

for all $\bar{u}, \bar{v} \in L^{2}\left(\Gamma_{C} ; \mathbb{R}^{d}\right)$ which entails $\left(\eta^{1}, \eta^{2}\right) \in \partial G(t, w, z, u, v)$. The above finishes the proof that the graph is closed. This argument completes the proof of the lemma.

In order to prove that the multifunction $F$ defined by (22) satisfies the hypothesis $H(F)_{1}$, we need additional conditions on the superpotentials $j_{k}$ for $k=1, \ldots, 4$.

$\underline{H\left(j_{1}\right)_{1}}: j_{1}: \Gamma_{C} \times(0, T) \times\left(\mathbb{R}^{d}\right)^{2} \times \mathbb{R} \rightarrow \mathbb{R}$ is such that

$$
\left|\partial j_{1}\left(x, t, \zeta_{1}, \rho_{1}, r_{1}\right)-\partial j_{1}\left(x, t, \zeta_{2}, \rho_{2}, r_{2}\right)\right| \leq L_{1}\left(\left\|\zeta_{1}-\zeta_{2}\right\|+\left\|\rho_{1}-\rho_{2}\right\|+\left|r_{1}-r_{2}\right|\right)
$$

for all $\zeta_{1}, \zeta_{2}, \rho_{1}, \rho_{2} \in \mathbb{R}^{d}, r_{1}, r_{2} \in \mathbb{R}$, a.e. $(x, t) \in \Gamma_{C} \times(0, T)$ with a constant $L_{1} \geq 0$. $H\left(j_{2}\right)_{1}: j_{2}: \Gamma_{C} \times(0, T) \times\left(\mathbb{R}^{d}\right)^{2} \times \mathbb{R} \rightarrow \mathbb{R}$ is such that

$$
\begin{aligned}
& \left(\partial j_{2}\left(x, t, \zeta_{1}, \rho_{1}, r_{1}\right)-\partial j_{2}\left(x, t, \zeta_{2}, \rho_{2}, r_{2}\right)\right)\left(r_{1}-r_{2}\right) \\
& \quad \geq-L_{2}\left(\left\|\zeta_{1}-\zeta_{2}\right\|+\left\|\rho_{1}-\rho_{2}\right\|+\left|r_{1}-r_{2}\right|\right)\left|r_{1}-r_{2}\right|
\end{aligned}
$$

for all $\zeta_{1}, \zeta_{2}, \rho_{1}, \rho_{2} \in \mathbb{R}^{d}, r_{1}, r_{2} \in \mathbb{R}$, a.e. $(x, t) \in \Gamma_{C} \times(0, T)$ with a constant $L_{2} \geq 0$. $\underline{H\left(j_{3}\right)_{1}}: j_{3}: \Gamma_{C} \times(0, T) \times\left(\mathbb{R}^{d}\right)^{3} \rightarrow \mathbb{R}$ is such that

$$
\left\|\partial j_{3}\left(x, t, \zeta_{1}, \rho_{1}, \theta_{1}\right)-\partial j_{3}\left(x, t, \zeta_{2}, \rho_{2}, \theta_{2}\right)\right\| \leq L_{3}\left(\left\|\zeta_{1}-\zeta_{2}\right\|+\left\|\rho_{1}-\rho_{2}\right\|+\left\|\theta_{1}-\theta_{2}\right\|\right)
$$

for all $\zeta_{1}, \zeta_{2}, \rho_{1}, \rho_{2}, \theta_{1}, \theta_{2} \in \mathbb{R}^{d}$, a.e. $(x, t) \in \Gamma_{C} \times(0, T)$ with a constant $L_{3} \geq 0$.

$\underline{H\left(j_{4}\right)_{1}}: j_{4}: \Gamma_{C} \times(0, T) \times\left(\mathbb{R}^{d}\right)^{3} \rightarrow \mathbb{R}$ is such that

$$
\begin{aligned}
& \left(\partial j_{4}\left(x, t, \zeta_{1}, \rho_{1}, \theta_{1}\right)-\partial j_{4}\left(x, t, \zeta_{2}, \rho_{2}, \theta_{2}\right), \theta_{1}-\theta_{2}\right) \\
& \quad \geq-L_{4}\left(\left\|\zeta_{1}-\zeta_{2}\right\|+\left\|\rho_{1}-\rho_{2}\right\|+\left\|\theta_{1}-\theta_{2}\right\|\right)\left\|\theta_{1}-\theta_{2}\right\|
\end{aligned}
$$

for all $\zeta_{1}, \zeta_{2}, \rho_{1}, \rho_{2}, \theta_{1}, \theta_{2} \in \mathbb{R}^{d}$, a.e. $(x, t) \in \Gamma_{C} \times(0, T)$ with a constant $L_{4} \geq 0$.

Remark 15 The hypothesis $H\left(j_{2}\right)_{1}$ (and $\left.H\left(j_{4}\right)_{1}\right)$ has been introduced and used earlier in [23] (under the name of relaxed monotonicity condition) in the case when $j_{2}$ (and $j_{4}$ ) does not depend on the variables $\zeta$ and $\rho$. 
Lemma 16 Assume that the hypotheses $H\left(j_{k}\right)$ hold for $k=1, \ldots, 4$, and that either

$$
\begin{aligned}
& j_{k}(x, t, \zeta, \rho, \cdot) \text { are regular and } j_{k} \text { satisfy } H\left(j_{k}\right)_{1} \text { for } k=1, \ldots, 4 \text { or } \\
& -j_{k}(x, t, \zeta, \rho, \cdot) \text { are regular and } \quad-j_{k} \text { satisfy } H\left(j_{k}\right)_{1} \text { for } k=1, \ldots, 4 \text {. }
\end{aligned}
$$

Then the multifunction $F:(0, T) \times V \times V \rightarrow 2^{Z^{*}}$ defined by (22) with the functional $G$ given by (19) and its integrand $g$ defined by (14), satisfies the condition $H(F)_{1}$ with $m_{2}=$ $c_{e} k_{1}\|\gamma\|^{2}$ and $m_{3}=c_{e} k_{2}\|\gamma\|^{2}$.

Proof It is clear that under the hypotheses, the condition $H(j)_{\text {reg }}$ holds. By Lemma 11 we know that the integrand $g$ given by (14) satisfies $H(g)$ and $H(g)_{\text {reg }}$. Hence by Lemma 12, it follows that the functional $G$ given by (19) satisfies $H(G)$. Using Lemma 14, under $H(G)$, we obtain that the multifunction $F$ satisfies $H(F)$.

Now, it is enough to prove that the multifunction $F$ satisfies $H(F)_{1}$ (iv). We suppose (24), the case when (25) holds can be treated analogously. We show that the following inequality holds:

$$
\begin{aligned}
& \left(\partial g\left(x, t, \xi_{1}, \eta_{1}, \xi_{1}, \eta_{1}\right)-\partial g\left(x, t, \xi_{2}, \eta_{2}, \xi_{2}, \eta_{2}\right),\left(\eta_{1}-\eta_{2}, \eta_{1}-\eta_{2}\right)\right)_{\mathbb{R}^{d} \times \mathbb{R}^{d}} \\
& \quad \geq-k_{1}\left\|\eta_{1}-\eta_{2}\right\|^{2}-k_{2}\left\|\eta_{1}-\eta_{2}\right\|\left\|\xi_{1}-\xi_{2}\right\|
\end{aligned}
$$

for all $\xi_{i}, \eta_{i} \in \mathbb{R}^{d}, i=1,2$, a.e. $(x, t) \in \Gamma_{C} \times(0, T)$ with $k_{1}, k_{2} \geq 0$. Under (24), it follows that $g(x, t, \zeta, \rho, \cdot, \cdot)$ is regular for all $\zeta, \rho \in \mathbb{R}^{d}$, a.e. $(x, t) \in \bar{\Gamma}_{C} \times(0, T)$. Using this regularity, by Theorem 2.3.10 of [6] and Proposition 5.6.33 of [7], we have

$$
\begin{aligned}
& \partial g(x, t, \zeta, \rho, \xi, \eta) \subset \partial_{\xi} g(x, t, \zeta, \rho, \xi, \eta) \times \partial_{\eta} g(x, t, \zeta, \rho, \xi, \eta) \\
&=\left(N_{1}^{*} \partial j_{1}\left(x, t, \zeta, \rho, N_{1} \xi\right)+N_{2}^{*} \partial j_{3}\left(x, t, \zeta, \rho, N_{2} \xi\right)\right) \\
& \times\left(N_{1}^{*} \partial j_{2}\left(x, t, \zeta, \rho, N_{1} \eta\right)+N_{2}^{*} \partial j_{4}\left(x, t, \zeta, \rho, N_{2} \eta\right)\right) \\
&=\left(\partial j_{1}\left(x, t, \zeta, \rho, \xi_{v}\right) v+\left(\partial j_{3}\left(x, t, \zeta, \rho, \xi_{\tau}\right)\right)_{\tau}\right) \\
& \times\left(\partial j_{2}\left(x, t, \zeta, \rho, \eta_{v}\right) v+\left(\partial j_{4}\left(x, t, \zeta, \rho, \eta_{\tau}\right)\right)_{\tau}\right)
\end{aligned}
$$

where $\partial g$ denotes the subdifferential of $g$ with respect to $(\xi, \eta), N_{1} \in \mathcal{L}\left(\mathbb{R}^{d}, \mathbb{R}\right), N_{2} \in$ $\mathcal{L}\left(\mathbb{R}^{d}, \mathbb{R}^{d}\right)$ are operators defined by $N_{1} \xi=\xi_{v}, N_{2} \xi=\xi_{\tau}$ for all $\xi \in \mathbb{R}^{d}$ with their adjoints $N_{1}^{*} \in \mathcal{L}\left(\mathbb{R}, \mathbb{R}^{d}\right), N_{2}^{*} \in \mathcal{L}\left(\mathbb{R}^{d}, \mathbb{R}^{d}\right)$ given by $N_{1}^{*} r=r v, N_{2}^{*} \xi=\xi_{\tau}$ for all $r \in \mathbb{R}, \xi \in \mathbb{R}^{d}$ i.e., $N_{2}^{*}=N_{2}$.

Let $\left(\bar{\chi}_{i}, \bar{\sigma}_{i}\right) \in \partial g\left(x, t, \xi_{i}, \eta_{i}, \xi_{i}, \eta_{i}\right),(x, t) \in \Gamma_{C} \times(0, T)$ with $\xi_{i}, \eta_{i} \in \mathbb{R}^{d}, i=1,2$. For simplicity of notation we omit the dependence on $(x, t)$. Then for $k=1,2$ we have $\bar{\chi}_{k} \in \partial j_{1}\left(x, t, \xi_{k}, \eta_{k}, \xi_{k v}\right) v+\left(\partial j_{3}\left(x, t, \xi_{k}, \eta_{k}, \xi_{k \tau}\right)\right)_{\tau}$ and $\bar{\sigma}_{k} \in \partial j_{2}\left(x, t, \xi_{k}, \eta_{k}, \eta_{k v}\right) v+$ $\left(\partial j_{4}\left(x, t, \xi_{k}, \eta_{k}, \eta_{k \tau}\right)\right)_{\tau}$ which means that

$$
\bar{\chi}_{1}=\alpha_{1} v+\gamma_{1 \tau}, \quad \bar{\sigma}_{1}=\beta_{1} v+\delta_{1 \tau}, \quad \bar{\chi}_{2}=\alpha_{2} v+\gamma_{2 \tau}, \quad \bar{\sigma}_{2}=\beta_{2} v+\delta_{2 \tau},
$$

with

$$
\begin{array}{ll}
\alpha_{i} \in \partial j_{1}\left(x, t, \xi_{i}, \eta_{i}, \xi_{i v}\right), & \beta_{i} \in \partial j_{2}\left(x, t, \xi_{i}, \eta_{i}, \eta_{i v}\right), \\
\gamma_{i} \in \partial j_{3}\left(x, t, \xi_{i}, \eta_{i}, \xi_{i \tau}\right), & \delta_{i} \in \partial j_{4}\left(x, t, \xi_{i}, \eta_{i}, \eta_{i \tau}\right)
\end{array}
$$


for $i=1,2$. By the hypotheses $H\left(j_{k}\right)_{1}$ for $k=1,3$, we have

$$
\begin{aligned}
& \left|\left(\partial j_{k}\left(x, t, \xi_{1}, \eta_{1}, \xi_{1 v}\right)-\partial j_{k}\left(x, t, \xi_{2}, \eta_{2}, \xi_{2 v}\right)\right)\left(\eta_{1 v}-\eta_{2 v}\right)\right| \\
& \quad \leq L_{k}\left(2\left\|\xi_{1}-\xi_{2}\right\|+\left\|\eta_{1}-\eta_{2}\right\|\right)\left\|\eta_{1}-\eta_{2}\right\|
\end{aligned}
$$

whereas for $k=2,4$ we have

$$
\begin{aligned}
& \left.\left(\partial j_{k}\left(x, t, \xi_{1}, \eta_{1}, \eta_{1 v}\right)-\partial j_{(} x, t, \xi_{2}, \eta_{2}, \eta_{2 v}\right)\right)\left(\eta_{1 v}-\eta_{2 v}\right) \\
& \quad \geq-L_{k}\left(\left\|\xi_{1}-\xi_{2}\right\|+2\left\|\eta_{1}-\eta_{2}\right\|\right)\left\|\eta_{1}-\eta_{2}\right\| .
\end{aligned}
$$

Using the last four inequalities and the fact that $\left(\zeta_{\tau}, \rho\right)_{\mathbb{R}^{d}}=\left(\zeta, \rho_{\tau}\right)_{\mathbb{R}^{d}}$ for all $\zeta, \rho \in \mathbb{R}^{d}$, we calculate

$$
\begin{aligned}
\left(\partial g\left(x, t, \xi_{1}, \eta_{1}, \xi_{1}, \eta_{1}\right)-\partial g\left(x, t, \xi_{2}, \eta_{2}, \xi_{2}, \eta_{2}\right),\left(\eta_{1}-\eta_{2}, \eta_{1}-\eta_{2}\right)\right)_{\mathbb{R}^{d} \times \mathbb{R}^{d}} \\
=\left(\bar{\chi}_{1}-\bar{\chi}_{2}, \eta_{1}-\eta_{2}\right)_{\mathbb{R}^{d}}+\left(\bar{\sigma}_{1}-\bar{\sigma}_{2}, \eta_{1}-\eta_{2}\right)_{\mathbb{R}^{d}} \\
=\left(\alpha_{1}-\alpha_{2}\right)\left(\eta_{1 v}-\eta_{2 v}\right)+\left(\beta_{1}-\beta_{2}\right)\left(\eta_{1 v}-\eta_{2 v}\right) \\
\quad+\left(\gamma_{1}-\gamma_{2}, \eta_{1 \tau}-\eta_{2 \tau}\right)_{\mathbb{R}^{d}}+\left(\delta_{1}-\delta_{2}, \eta_{1 \tau}-\eta_{2 \tau}\right)_{\mathbb{R}^{d}} \\
\geq \\
\geq
\end{aligned}
$$

with $k_{1}=\max \left\{L_{1}, 2 L_{2}, L_{3}, 2 L_{4}\right\}$ and $k_{2}=\max \left\{2 L_{1}, L_{2}, 2 L_{3}, L_{4}\right\}$. Hence the proof of the property (26) is complete.

Next we will prove that the subdifferential $\partial G$ of the functional $G$ defined by (19) satisfies the condition

$$
\begin{aligned}
& \left\langle\partial G\left(t, w_{1}, z_{1}, w_{1}, z_{1}\right)-\partial G\left(t, w_{2}, z_{2}, w_{2}, z_{2}\right),\left(z_{1}-z_{2}, z_{1}-z_{2}\right)\right\rangle_{L^{2}\left(\Gamma_{C} ; \mathbb{R}^{d}\right)^{2}} \\
& \quad \geq-k_{1}\left\|z_{1}-z_{2}\right\|_{L^{2}\left(\Gamma_{C} ; \mathbb{R}^{d}\right)}^{2}-k_{2}\left\|z_{1}-z_{2}\right\|_{L^{2}\left(\Gamma_{C} ; \mathbb{R}^{d}\right)}\left\|w_{1}-w_{2}\right\|_{L^{2}\left(\Gamma_{C} ; \mathbb{R}^{d}\right)}
\end{aligned}
$$

for all $w_{i}, z_{i} \in L^{2}\left(\Gamma_{C} ; \mathbb{R}^{d}\right)$, a.e. $t \in(0, T)$ with $k_{1}, k_{2} \geq 0$, where $\partial G$ denotes the subdifferential of $G(t, w, z, \cdot, \cdot)$. Similarly as in the proof of Lemma 12 and Theorem 2.7.5 of Clarke [6], we use the property that if $(\bar{u}, \bar{v}) \in \partial G(t, w, z, u, v)$ for a.e. $t \in(0, T)$ then

$$
(\bar{u}(x), \bar{v}(x)) \in \partial g(x, t, w(x), z(x), u(x), v(x)) \quad \text { for a.e. }(x, t) \in \Gamma_{C} \times(0, T),
$$

for every $w, z, u, v, \bar{u}, \bar{v} \in L^{2}\left(\Gamma_{C} ; \mathbb{R}^{d}\right)$. For the proof of (27), let $w_{i}, z_{i}, \bar{u}_{i}, \bar{v}_{i} \in L^{2}\left(\Gamma_{C} ; \mathbb{R}^{d}\right)$ with $\left(\bar{u}_{i}, \bar{v}_{i}\right) \in \partial G\left(t, w_{i}, z_{i}, w_{i}, z_{i}\right)$ for $i=1,2$, a.e. $t \in(0, T)$. From the aforementioned property, we know that

$$
\left(\bar{u}_{i}(x), \bar{v}_{i}(x)\right) \in \partial g\left(x, t, w_{i}(x), z_{i}(x), w_{i}(x), z_{i}(x)\right)
$$

for a.e. $(x, t) \in \Gamma_{C} \times(0, T)$. Exploiting the inequality (26), we have

$$
\begin{aligned}
& \left(\left(\bar{u}_{1}(x), \bar{v}_{1}(x)\right)-\left(\bar{u}_{2}(x), \bar{v}_{2}(x)\right),\left(z_{1}(x)-z_{2}(x), z_{1}(x)-z_{2}(x)\right)\right)_{\mathbb{R}^{d} \times \mathbb{R}^{d}} \\
& \quad \geq-k_{1}\left\|z_{1}(x)-z_{2}(x)\right\|^{2}-k_{2}\left\|w_{1}(x)-w_{2}(x)\right\|\left\|z_{1}(x)-z_{2}(x)\right\|
\end{aligned}
$$

for a.e. $x \in \Gamma_{C}$. Integrating this inequality over $\Gamma_{C}$ and applying the Hölder inequality, we obtain 


$$
\begin{aligned}
& \left\langle\left(\bar{u}_{1}, \bar{v}_{1}\right)-\left(\bar{u}_{2}, \bar{v}_{2}\right),\left.\left(z_{1}-z_{2}, z_{1}-z_{2}\right)\right|_{L^{2}\left(\Gamma_{C} ; \mathbb{R}^{d}\right)^{2}}\right. \\
& \quad=\int_{\Gamma_{C}}\left(\left(\bar{u}_{1}(x)-\bar{u}_{2}(x)\right) \cdot\left(z_{1}(x)-z_{2}(x)\right)+\left(\bar{v}_{1}(x)-\bar{v}_{2}(x)\right) \cdot\left(z_{1}(x)-z_{2}(x)\right)\right) d \Gamma \\
& \quad \geq-k_{1}\left\|z_{1}-z_{2}\right\|_{L^{2}\left(\Gamma_{C} ; \mathbb{R}^{d}\right)}^{2}-k_{2}\left\|z_{1}-z_{2}\right\|_{L^{2}\left(\Gamma_{C} ; \mathbb{R}^{d}\right)}\left\|w_{1}-w_{2}\right\|_{L^{2}\left(\Gamma_{C} ; \mathbb{R}^{d}\right)},
\end{aligned}
$$

which means that (27) is satisfied.

Finally we show that the multifunction $F$ defined by (22) satisfies $H(F)_{1}(\mathrm{iv})$. Let $u_{i}$, $v_{i} \in V, t \in(0, T)$ and $z_{i} \in F\left(t, u_{i}, v_{i}\right)$ for $i=1,2$. By the definition of $F$, we have

$$
\begin{aligned}
& z_{1}=a_{1}+a_{2}, \quad\left(a_{1}, a_{2}\right)=R^{*}\left(\eta_{1}, \eta_{2}\right)=\left(\gamma^{*} \eta_{1}, \gamma^{*} \eta_{2}\right), \\
& \quad\left(\eta_{1}, \eta_{2}\right) \in \partial G\left(t, \gamma u_{1}, \gamma v_{1}, \gamma u_{1}, \gamma v_{1}\right), \\
& z_{2}=b_{1}+b_{2}, \quad\left(b_{1}, b_{2}\right)=R^{*}\left(\xi_{1}, \xi_{2}\right)=\left(\gamma^{*} \xi_{1}, \gamma^{*} \xi_{2}\right), \\
& \quad\left(\xi_{1}, \xi_{2}\right) \in \partial G\left(t, \gamma u_{2}, \gamma v_{2}, \gamma u_{2}, \gamma v_{2}\right),
\end{aligned}
$$

with $a_{i}, b_{i} \in Z^{*}$ and $\eta_{i}, \xi_{i} \in L^{2}\left(\Gamma_{C} ; \mathbb{R}^{d}\right), i=1,2$. Exploiting (27) and the continuity of the trace operator, we obtain

$$
\begin{aligned}
& \left\langle z_{1}-z_{2}, v_{1}-v_{2}\right\rangle_{Z^{*} \times Z}=\left\langle a_{1}+a_{2}-b_{1}-b_{2}, v_{1}-v_{2}\right\rangle_{Z^{*} \times Z} \\
& \quad=\left\langle\left(\eta_{1}, \eta_{2}\right)-\left(\xi_{1}, \xi_{2}\right),\left(\gamma v_{1}-\gamma v_{2}, \gamma v_{1}-\gamma v_{2}\right)\right\rangle_{L^{2}\left(\Gamma_{C} ; \mathbb{R}^{d}\right)^{2}} \\
& \geq-k_{1} c_{e}\|\gamma\|^{2}\left\|v_{1}-v_{2}\right\|^{2}-k_{2} c_{e}\|\gamma\|^{2}\left\|v_{1}-v_{2}\right\|\left\|u_{1}-u_{2}\right\|,
\end{aligned}
$$

where $c_{e}>0$ is the embedding constant of $V$ into $Z$ and $\|\gamma\|$ is the norm of the trace operator. Thus the condition $H(F)_{1}$ (iv) holds with $m_{2}=c_{e} k_{1}\|\gamma\|^{2}$ and $m_{3}=c_{e} k_{2}\|\gamma\|^{2}$. The proof of the lemma is complete.

In order to formulate and prove the results on the existence and uniqueness of solutions to the hemivariational inequality in Problem (HVI), we need the following two lemmas.

Lemma 17 Under hypotheses $H(\mathcal{A}), H(\mathcal{B}), H(\mathcal{C}), H(f)$ and $H\left(j_{k}\right)$ for $k=1, \ldots, 4$, every solution of the Problem $\mathcal{P}$ with the multivalued mapping of the form $(22)$, with $G:(0, T) \times$ $L^{2}\left(\Gamma_{C} ; \mathbb{R}^{d}\right)^{4} \rightarrow \mathbb{R}$ of the form (19) and its integrand $g: \Gamma_{C} \times(0, T) \times\left(\mathbb{R}^{d}\right)^{4} \rightarrow \mathbb{R}$ given by (14), and the operators $A, B$ and $C$ defined by (9), (10) and (11) respectively, is a solution to Problem (HVI).

Proof Let $u \in \mathcal{V}$ with $u^{\prime} \in \mathcal{W}$ be a solution of Problem $\mathcal{P}$. Then there exists $z \in \mathcal{Z}^{*}$ such that

$$
\begin{aligned}
& u^{\prime \prime}(t)+A\left(t, u^{\prime}(t)\right)+B(t, u(t))+\int_{0}^{t} C(t-s) u(s) d s+z(t)=f(t) \quad \text { a.e. } t \\
& z(t) \in S R^{*} \partial G\left(t, R\left(u(t), u^{\prime}(t)\right), R\left(u(t), u^{\prime}(t)\right)\right) \quad \text { a.e. } t \in(0, T) \\
& u(0)=u_{0}, \quad u^{\prime}(0)=u_{1} .
\end{aligned}
$$

Hence, by the definition of the multivalued term, we obtain $z(t)=z_{1}(t)+z_{2}(t)$, $\left(z_{1}(t), z_{2}(t)\right)=\left(\gamma^{*} \eta_{1}(t), \gamma^{*} \eta_{2}(t)\right)$ and $\left(\eta_{1}(t), \eta_{2}(t)\right) \in \partial G\left(t, \gamma u(t), \gamma u^{\prime}(t), \gamma u(t), \gamma u^{\prime}(t)\right)$ 
for a.e. $t \in(0, T)$, where $\eta_{i} \in L^{2}\left(0, T ; L^{2}\left(\Gamma_{C} ; \mathbb{R}^{d}\right)\right), i=1,2$. The last inclusion, by the definition of the subdifferential is equivalent to

$$
\left\langle\eta_{1}(t), \bar{u}\right\rangle_{L^{2}\left(\Gamma_{C} ; \mathbb{R}^{d}\right)}+\left\langle\eta_{2}(t),\left.\bar{v}\right|_{L^{2}\left(\Gamma_{C} ; \mathbb{R}^{d}\right)} \leq G^{0}\left(t, \gamma u(t), \gamma u^{\prime}(t), \gamma u(t), \gamma u^{\prime}(t) ; \bar{u}, \bar{v}\right)\right.
$$

for all $\bar{u}, \bar{v} \in L^{2}\left(\Gamma_{C} ; \mathbb{R}^{d}\right)$ and a.e. $t \in(0, T)$. On the other hand, by Lemmas 11 and 12, we have

$$
\begin{aligned}
& G^{0}\left(t, \gamma u(t), \gamma u^{\prime}(t), \gamma u(t), \gamma u^{\prime}(t) ; \bar{u}, \bar{v}\right) \\
& =\int_{\Gamma_{C}}\left(j_{1}^{0}\left(x, t, u(t), u^{\prime}(t), u_{v}(t) ; \bar{u}_{v}\right)+j_{2}^{0}\left(x, t, u(t), u^{\prime}(t), u_{v}^{\prime}(t) ; \bar{v}_{v}\right)\right. \\
& \left.\quad+j_{3}^{0}\left(x, t, u(t), u^{\prime}(t), u_{\tau}(t) ; \bar{u}_{\tau}\right)+j_{4}^{0}\left(x, t, u(t), u^{\prime}(t), u_{\tau}^{\prime}(t) ; \bar{v}_{\tau}\right)\right) d \Gamma
\end{aligned}
$$

for all $\bar{u}, \bar{v} \in L^{2}\left(\Gamma_{C} ; \mathbb{R}^{d}\right)$, a.e. $t \in(0, T)$. By (28), (29) and (30), for all $v \in V$ and a.e. $t \in(0, T)$, we deduce

$$
\begin{aligned}
& \left\langle f(t)-u^{\prime \prime}(t)-A\left(t, u^{\prime}(t)\right)-B(t, u(t))-\int_{0}^{t} C(t-s) u(s) d s, v\right\rangle \\
& =\langle z(t), v\rangle_{Z^{*} \times Z}=\left\langle\eta_{1}(t), \gamma v\right\rangle_{L^{2}\left(\Gamma_{C} ; \mathbb{R}^{d}\right)}+\left\langle\eta_{2}(t), \gamma v\right\rangle_{L^{2}\left(\Gamma_{C} ; \mathbb{R}^{d}\right)} \\
& \leq \int_{\Gamma_{C}}\left(j_{1}^{0}\left(x, t, u(t), u^{\prime}(t), u_{v}(t) ; v_{v}\right)+j_{2}^{0}\left(x, t, u(t), u^{\prime}(t), u_{v}^{\prime}(t) ; v_{v}\right)\right. \\
& \left.\quad+j_{3}^{0}\left(x, t, u(t), u^{\prime}(t), u_{\tau}(t) ; v_{\tau}\right)+j_{4}^{0}\left(x, t, u(t), u^{\prime}(t), u_{\tau}^{\prime}(t) ; v_{\tau}\right)\right) d \Gamma,
\end{aligned}
$$

which means that $u$ is a solution to Problem (HVI). The proof of the lemma is complete.

Lemma 18 Assume the function definitions and hypotheses of Lemma 17. If either $j_{1}=$ $j_{3}=0$ or $j_{2}=j_{4}=0$, then $u$ is a solution to Problem (HVI) if and only if $u$ is a solution to Problem $\mathcal{P}$.

Proof In view of Lemma 17, it is enough to show that every solution to Problem (HVI) is a solution to Problem $\mathcal{P}$. Let $u \in \mathcal{V}$ with $u^{\prime} \in \mathcal{W}$ be a solution of Problem (HVI), i.e., $u(0)=u_{0}, u^{\prime}(0)=u_{1}$ and

$$
\begin{aligned}
& \left\langle f(t)-u^{\prime \prime}(t)-A\left(t, u^{\prime}(t)\right)-B(t, u(t))-\int_{0}^{t} C(t-s) u(s) d s, v\right\rangle \\
& \leq \int_{\Gamma_{C}}\left(j_{1}^{0}\left(x, t, u(t), u^{\prime}(t), u_{v}(t) ; v_{v}\right)+j_{2}^{0}\left(x, t, u(t), u^{\prime}(t), u_{v}^{\prime}(t) ; v_{v}\right)\right. \\
& \left.\quad+j_{3}^{0}\left(x, t, u(t), u^{\prime}(t), u_{\tau}(t) ; v_{\tau}\right)+j_{4}^{0}\left(x, t, u(t), u^{\prime}(t), u_{\tau}^{\prime}(t) ; v_{\tau}\right)\right) d \Gamma
\end{aligned}
$$

for all $v \in V$, a.e. $t \in(0, T)$, where the operators $A, B$ and $C$ are defined by (9), (10) and (11), respectively. From $H(j)_{\text {reg }}$, by Lemmas 11 and 12, we know that equalities (15) and (20) hold, which implies 


$$
\begin{aligned}
\int_{\Gamma_{C}}\left(j_{1}^{0}\left(x, t, u(t), u^{\prime}(t), u_{v}(t) ; v_{v}\right)+j_{2}^{0}\left(x, t, u(t), u^{\prime}(t), u_{v}^{\prime}(t) ; v_{v}\right)\right. \\
\left.\quad+j_{3}^{0}\left(x, t, u(t), u^{\prime}(t), u_{\tau}(t) ; v_{\tau}\right)+j_{4}^{0}\left(x, t, u(t), u^{\prime}(t), u_{\tau}^{\prime}(t) ; v_{\tau}\right)\right) d \Gamma \\
=G^{0}\left(t, \gamma u(t), \gamma u^{\prime}(t), \gamma u(t), \gamma u^{\prime}(t) ; \gamma v, \gamma v\right)
\end{aligned}
$$

for all $v \in V$, a.e. $t \in(0, T)$. Suppose now that $j_{1}=j_{3}=0$. Then $g$ is given by $g(x, t, \zeta, \rho, \xi, \eta)=j_{2}\left(x, t, \zeta, \rho, \eta_{v}\right)+j_{4}\left(x, t, \zeta, \rho, \eta_{\tau}\right)$ for all $\zeta, \rho, \xi, \eta \in \mathbb{R}^{d}$, a.e. $(x, t) \in$ $\Gamma_{C} \times(0, T)$ and is independent of $\xi$, and consequently $G$ is given by

$$
G(t, \widehat{w}, \widehat{z}, \widehat{u}, \widehat{v})=\int_{\Gamma_{C}}\left(j_{2}\left(x, t, \widehat{w}(x), \widehat{z}(x), \widehat{v}_{v}(x)\right)+j_{4}\left(x, t, \widehat{w}(x), \widehat{z}(x), \widehat{v}_{\tau}(x)\right)\right) d \Gamma
$$

for $\widehat{w}, \widehat{z}, \widehat{u}, \widehat{v} \in L^{2}\left(\Gamma_{C} ; \mathbb{R}^{d}\right)$, a.e. $t \in(0, T)$ and is independent of $\widehat{u}$. We denote the latter by $G_{1}$, i.e.,

$$
G(t, \widehat{w}, \widehat{z}, \widehat{u}, \widehat{v})=G_{1}(t, \widehat{w}, \widehat{z}, \widehat{v}) \quad \text { for } \widehat{w}, \widehat{z}, \widehat{u}, \widehat{v} \in L^{2}\left(\Gamma_{C} ; \mathbb{R}^{d}\right) \text {, a.e. } t \in(0, T),
$$

with $G_{1}:(0, T) \times L^{2}\left(\Gamma_{C} ; \mathbb{R}^{d}\right)^{3} \rightarrow \mathbb{R}$. We observe that

$$
G^{0}(t, \widehat{w}, \widehat{z}, \widehat{u}, \widehat{v} ; \bar{u}, \bar{v})=G_{1}^{0}(t, \widehat{w}, \widehat{z}, \widehat{v} ; \bar{v})
$$

for all $\widehat{w}, \widehat{z}, \widehat{u}, \widehat{v}, \bar{u}, \bar{v} \in L^{2}\left(\Gamma_{C} ; \mathbb{R}^{d}\right)$, a.e. $t \in(0, T)$, where $G_{1}^{0}$ denotes the generalized derivative of $G_{1}(t, \widehat{w}, \widehat{z}, \cdot)$, and

$$
\partial G(t, \widehat{w}, \widehat{z}, \widehat{u}, \widehat{v})=\{0\} \times \partial G_{1}(t, \widehat{w}, \widehat{z}, \widehat{v})
$$

for all $\widehat{w}, \widehat{z}, \widehat{u}, \widehat{v} \in L^{2}\left(\Gamma_{C} ; \mathbb{R}^{d}\right)$, a.e. $t \in(0, T)$, where $\partial G_{1}$ denotes the generalized gradient of $G_{1}(t, \widehat{w}, \widehat{z}, \cdot)$. From (31)-(34), we obtain

$$
\begin{aligned}
& \left\langle f(t)-u^{\prime \prime}(t)-A\left(t, u^{\prime}(t)\right)-B(t, u(t))-\int_{0}^{t} C(t-s) u(s) d s, v\right\rangle \\
& \quad \leq G_{1}^{0}\left(t, \gamma u(t), \gamma u^{\prime}(t), \gamma u^{\prime}(t) ; \gamma v\right)
\end{aligned}
$$

for all $v \in V$, a.e. $t \in(0, T)$. Using the equality

$$
G_{1}^{0}\left(t, \gamma u(t), \gamma u^{\prime}(t), \gamma u^{\prime}(t) ; \gamma v\right)=\left(G_{1} \circ \gamma\right)^{0}\left(t, \gamma u(t), \gamma u^{\prime}(t), u^{\prime}(t) ; v\right)
$$

(which is a consequence of Theorem 2.3.10 of [6] and the regularity of $G_{1}(t, \widehat{w}, \widehat{z}, \cdot)$ ), from (36), it follows that

$$
\begin{aligned}
& f(t)-u^{\prime \prime}(t)-A\left(t, u^{\prime}(t)\right)-B(t, u(t))-\int_{0}^{t} C(t-s) u(s) d s \\
& \quad \in \partial\left(G_{1} \circ \gamma\right)\left(t, \gamma u(t), \gamma u^{\prime}(t), u^{\prime}(t)\right)=\gamma^{*} \partial G_{1}\left(t, \gamma u(t), \gamma u^{\prime}(t), \gamma u^{\prime}(t)\right)
\end{aligned}
$$

for a.e. $t \in(0, T)$. The last equality follows from Theorem 2.3 .10 of [6].

On the other hand, we observe that the multifunction $F$ defined by (22), with $G$ given by (33), is now of the form 


$$
\begin{aligned}
F(t, \tilde{u}, v) & =S R^{*} \partial G(t, R(\tilde{u}, v), R(\widetilde{u}, v))=S R^{*}\left(\{0\} \times \partial G_{1}(t, R(\tilde{u}, v), \gamma v)\right) \\
& =S\left(\{0\}, \gamma^{*} \partial G_{1}(t, R(\tilde{u}, v), \gamma v)\right)=\gamma^{*} \partial G_{1}(t, \gamma \tilde{u}, \gamma v, \gamma v)
\end{aligned}
$$

for all $\tilde{u}, v \in V$, a.e. $t \in(0, T)$. Therefore, from (37), we have

$$
f(t)-u^{\prime \prime}(t)-A\left(t, u^{\prime}(t)\right)-B(t, u(t))-\int_{0}^{t} C(t-s) u(s) d s \in F\left(t, u(t), u^{\prime}(t)\right)
$$

for a.e. $t \in(0, T)$ which means that $u$ is a solution Problem $\mathcal{P}$.

The case when $j_{2}=j_{4}=0$ can be treated in an analogous way. This completes the proof of the lemma.

The following are the existence result for the hemivariational inequality in Problem (HVI) which are the direct conclusion from the lemmas above and Theorem 7.

Theorem 19 Under the hypotheses $H(\mathcal{A}), H(\mathcal{B}), H(\mathcal{C}), H(f), H\left(j_{k}\right)$ for $k=1, \ldots, 4$, either (24) or (25), and the following conditions

$$
\tilde{a}_{3}>4 \sqrt{15} c_{e}^{2}\|\gamma\|^{2}\left(T \max \left\{\max _{1 \leq k \leq 4} c_{k 1}, c_{13}, c_{33}\right\}+\max \left\{\max _{1 \leq k \leq 4} c_{k 2}, c_{23}, c_{43}\right\}\right)
$$

and

$$
\tilde{a}_{4}>c_{e}\|\gamma\|^{2}\left(\max \left\{L_{1}, 2 L_{2}, L_{3}, 2 L_{4}\right\}+\frac{T}{\sqrt{2}} \max \left\{2 L_{1}, L_{2}, 2 L_{3}, L_{4}\right\}\right),
$$

Problem (HVI) admits a solution.

Theorem 20 Assume the hypotheses of Theorem 19. If, in addition, either $j_{1}=j_{3}=0$ or $j_{2}=j_{4}=0$, then the hemivariational inequality in Problem (HVI) admits a unique solution.

\section{Applications to Viscoelastic Mechanical Problems}

The aim of this section is to explain, by providing several examples, formulations of multivalued boundary conditions of mechanics. We consider boundary conditions resulting from convex or nonconvex and nonsmooth potentials using the concept of a subdifferential. We restrict ourselves to one-dimensional examples, referring to Chap. 4.6 of [30] for two- and three-dimensional contact laws. We present specific examples of contact and friction laws which can be met in mechanics and which lead to the subdifferential boundary conditions of the form

$$
\begin{aligned}
& -\sigma_{v}(t) \in \partial j_{1}\left(x, t, u(t), u^{\prime}(t), u_{v}(t)\right)+\partial j_{2}\left(x, t, u(t), u^{\prime}(t), u_{v}^{\prime}(t)\right), \\
& -\sigma_{\tau}(t) \in \partial j_{3}\left(x, t, u(t), u^{\prime}(t), u_{\tau}(t)\right)+\partial j_{4}\left(x, t, u(t), u^{\prime}(t), u_{\tau}^{\prime}(t)\right)
\end{aligned}
$$

on $\Gamma_{C} \times(0, T)$. 


\subsection{Prescribed Normal Stress and Nonmonotone Friction Laws}

Let us consider the following boundary conditions on $\Gamma_{C} \times(0, T)$ :

$$
\begin{aligned}
& -\sigma_{v}(t)=S(t), \\
& -\sigma_{\tau}(t) \in \partial j_{4}\left(x, t, u(t), u^{\prime}(t), u_{\tau}^{\prime}(t)\right) .
\end{aligned}
$$

Equation (40) states that the normal stress is prescribed on $\Gamma_{C} \times(0, T)$ and is given by $S=S(x, t) \geq 0$. Such a condition makes sense when the real contact area is close to the nominal one and the surfaces are conforming. Then $S=S(x, t)$ is the contact pressure and it is given by the ratio of the total applied force to the nominal contact area. It is considered (see Chaps. 2.6 and 10.1 of Shillor et al. [41]) to be a good approximation when the load is light and the contact surface is transmitted by the asperity tips only. This law is of the form (38) with $j_{1}(x, t, \zeta, \rho, r)=S(x, t) r$ and $j_{2}=0$, where $S \in L^{\infty}\left(\Gamma_{C} \times(0, T)\right), S \geq 0$ is a given normal stress. It is clear that $j_{1}(x, t, \zeta, \rho, \cdot)$ is convex (hence regular), and that $H\left(j_{1}\right)$ and $H\left(j_{1}\right)_{1}$ hold.

\subsubsection{Nonmonotone Friction Independent of Slip and Slip Rate}

We consider the nonmonotone friction laws which are independent of the slip displacement and the slip rate. This is the case when the superpotential $j_{4}=j_{4}(x, t, \zeta, \rho, \theta)$ is independent of $(\zeta, \rho)$ and nonconvex in $\theta$. Then the friction law (41) takes the form

$$
-\sigma_{\tau}(t) \in \partial j_{4}\left(x, t, u_{\tau}^{\prime}(t)\right) \quad \text { on } \Gamma_{C} \times(0, T) .
$$

This law appears (cf. Sect. 7.2 of Panagiotopoulos [34]) in the tangential direction of the adhesive interface and describes the partial cracking and crushing of the adhesive bonding material. Several examples of zig-zag friction laws from Sect. 2.4 of Panagiotopoulos [34] can be formulated in the form (42). For instance, let $j_{4}: \mathbb{R} \rightarrow \mathbb{R}$ be given by $j_{4}(r)=\min \left\{\varphi_{1}(r), \varphi_{2}(r)\right\}$, where $\varphi_{1}(r)=a r^{2}, \varphi_{2}(r)=\frac{a}{2}\left(r^{2}+1\right), r \in \mathbb{R}$ (for simplicity we also drop the $(x, t)$-dependence) and $a>0$. Its subdifferential is as follows:

$$
\partial j_{4}(r)= \begin{cases}a r & r \in(-\infty,-1) \cup(1,+\infty), \\ 2 a r & r \in(-1,1), \\ {[a, 2 a]} & r=1, \\ {[-2 a,-a]} & r=-1 .\end{cases}
$$

By Theorem 2.5.1 of [6], we know that $\partial j_{4}(r) \subset \operatorname{co}\left\{\varphi_{1}^{\prime}(r), \varphi_{2}^{\prime}(r)\right\}$ and that the subdifferential $\partial j_{4}$ has at most linear growth. Since $j_{4}$ is the minimum of the strictly differentiable functions, the function $-j_{4}$ is regular. From the above and Proposition 2.1.1 of [6], the hypothesis $H\left(j_{4}\right)$ holds.

Another example of nonmonotone friction law can be obtained from the nonconvex function $j_{4}: \mathbb{R}^{d} \rightarrow \mathbb{R}$ given by

$$
j_{4}(\xi)= \begin{cases}\|\xi\|^{2} & \text { if }\|\xi\| \leq M \\ M^{2} & \text { if }\|\xi\|>M\end{cases}
$$


for $\xi \in \mathbb{R}^{d}$, where $M$ is a positive constant. This function can be represented as a difference of convex functions, i.e., $j_{4}(\xi)=\varphi_{1}(\xi)-\varphi_{2}(\xi)$ for $\xi \in \mathbb{R}^{d}$, where $\varphi_{1}(\xi)=\|\xi\|^{2}$ and

$$
\varphi_{2}(\xi)= \begin{cases}0 & \text { if }\|\xi\| \leq M, \\ \|\xi\|^{2}-M^{2} & \text { if }\|\xi\|>M .\end{cases}
$$

Since $\partial \varphi_{1}(\xi)$ is a singleton for $\xi \in \mathbb{R}^{d}$, by Lemma 14 of [29], we deduce that $-j_{4}$ is regular and $\partial j_{4}(\xi)=\partial \varphi_{1}(\xi)-\partial \varphi_{2}(\xi)$ for $\xi \in \mathbb{R}^{d}$. Additionally, it is easy to observe that $j_{4}$ satisfies $H\left(j_{4}\right)$.

\subsubsection{Contact with Nonmonotone Normal Damped Response}

This contact condition is of the form (38) with $j_{1}=0$ and it models the situations with granular or wet surfaces in which the response of the foundation depends on the normal velocity of the body. For simplicity, we describe the case when

$$
-\sigma_{v}(t) \in \partial j_{2}\left(u_{v}^{\prime}(t)\right) \quad \text { on } \Gamma_{C} \times(0, T) .
$$

The specific example of the nonmonotone normal damped response condition is given by the following nonconvex, regular and d.c. function

$$
j_{2}(r)=\left\{\begin{array}{ll}
0 & \text { if } r<0, \\
-\frac{1}{2} r^{2}+r & \text { if } 0 \leq r<1, \\
\frac{1}{2} & \text { if } r \geq 1,
\end{array} \quad \partial j_{2}(r)= \begin{cases}0 & \text { if } r<0, \\
{[0,1]} & \text { if } r=0, \\
-r+1 & \text { if } 0<r<1 \\
0 & \text { if } r \geq 1\end{cases}\right.
$$

It is clear that $\left|\partial j_{2}(r)\right| \leq 1+|r|$ for $r \in \mathbb{R}$; The function $j_{2}$ can be represented as the difference of convex functions, i.e. $j_{2}(r)=\varphi_{1}(r)-\varphi_{2}(r), r \in \mathbb{R}$, where

$$
\varphi_{1}(r)=\left\{\begin{array}{ll}
\frac{1}{2} r^{2}-r+1 & \text { if } r<0, \\
1 & \text { if } 0 \leq r<1, \\
\frac{1}{2} r^{2}-r+\frac{3}{2} & \text { if } r \geq 1,
\end{array} \quad \varphi_{2}(r)=\frac{1}{2} r^{2}-r+1 .\right.
$$

Since $\varphi_{1}, \varphi_{2}$ are convex functions, $\partial \varphi_{1}, \partial \varphi_{2}$ have a sublinear growth with $\partial \varphi_{2}$ being a singleton, we deduce by Lemma 14 of [29] that $j_{2}$ is regular. From the above and the Proposition 2.1.1 of [6], it is obvious that $H\left(j_{2}\right)$ holds. Next, we verify that $\eta_{1} \leq \eta_{2}-\left(r_{1}-r_{2}\right)$ for all $r_{1}<r_{2}$ and $\eta_{i} \in \partial j_{2}\left(r_{i}\right), i=1,2$ which implies relaxed monotonicity condition $\left(\partial j_{2}\left(r_{1}\right)-\partial j_{2}\left(r_{2}\right)\right)\left(r_{1}-r_{2}\right) \geq-\left|r_{1}-r_{2}\right|^{2}$ (cf. Remark 15), as well as $H\left(j_{2}\right)_{1}$.

\subsection{Viscous Contact with Tresca's Friction Law}

We consider a model of damped response contact with time-dependent Tresca's friction law. In this model the contact is characterized by the following boundary conditions:

$$
\left\{\begin{array}{l}
-\sigma_{v}(t)=k(x)\left|u_{v}^{\prime}(t)\right|^{q-1} u_{v}^{\prime}(t), \\
\left\|\sigma_{\tau}(t)\right\| \leq \psi(t) \quad \text { with } \\
\left\|\sigma_{\tau}(t)\right\|<\psi(t) \quad \Rightarrow \quad u_{\tau}^{\prime}(t)=0, \\
\left\|\sigma_{\tau}(t)\right\|=\psi(t) \quad \Rightarrow \quad \exists \lambda \geq 0: \sigma_{\tau}(t)=-\lambda u_{\tau}^{\prime}(t)
\end{array}\right.
$$


on $\Gamma_{C} \times(0, T)$, where $k \in L^{\infty}\left(\Gamma_{C}\right), k>0$ a.e. on $\Gamma_{C}, 0<q \leq 1, \psi \in L^{\infty}\left(\Gamma_{C} \times(0, T)\right)$ and $\psi \geq 0$ a.e. on $\Gamma_{C} \times(0, T)$, cf. Shillor and Sofonea [40] and Chap. 13 of Han and Sofonea [11]. These boundary conditions are of the form (38) and (39) with $j_{1}=j_{3}=0$, $j_{2}(x, t, \zeta, \rho, r)=\frac{k(x)}{q+1}|r|^{q+1}$ and $j_{4}(x, t, \zeta, \rho, \theta)=\psi(x, t)\|\theta\|$. Therefore

$$
\begin{aligned}
& \partial j_{2}(x, t, \zeta, \rho, r)=k(x)|r|^{q-1} r, \\
& \partial j_{4}(x, t, \zeta, \rho, \theta)=\psi(x, t) \partial\|\theta\|= \begin{cases}\psi(x, t) \bar{B}(0,1) & \text { if } \eta=0, \\
\psi(x, t) \frac{\eta}{\|\eta\|} & \text { if } \eta \neq 0 .\end{cases}
\end{aligned}
$$

Thus $H\left(j_{2}\right)_{1}$ holds with $c_{20}=c_{23}=\left\|k_{0}\right\|_{L^{\infty}\left(\Gamma_{C}\right)}, c_{21}=c_{22}=0$ while $j_{4}$ satisfies $H\left(j_{4}\right)_{1}$ with $c_{40}=\|\psi\|_{L^{\infty}\left(\Gamma_{C} \times(0, T)\right)}, c_{41}=c_{42}=c_{43}=0$ and $H\left(j_{4}\right)_{2} ; j_{4}$ is also convex (so regular) in $\theta$ and $H\left(j_{4}\right)_{3}$ holds. Classically the Tresca friction law is characterized by a given constant friction bound, that is, $\psi(x, t)=$ const., cf., e.g., Amassad and Fabre [1], Amassad and Sofonea [2, 3], Duvaut and Lions [10], Han and Sofonea [11], Panagiotopoulos [33], Selmani and Sofonea [40].

\subsection{Other Nonmonotone Friction Contact Laws}

In this part we comment on the boundary conditions expressed in the form

$$
-\sigma_{\tau}(t) \in \partial j_{3}\left(x, t, u(t), u^{\prime}(t), u_{\tau}(t)\right)
$$

This relation describes the tangential contact law between reinforcement and concrete in a concrete structure. In literature, cf. Chap. 2.4 in Panagiotopoulos [34] (diagrams of Fig. 2.4.1), Chap. 1.4 in Naniewicz and Panagiotopoulos [30] (diagrams of Fig. 1.4.3), one can find a couple of examples of the superpotential $j_{3}$ which describes such type of contact. We give two examples of nonconvex functions which appear in (43).

In the first example the superpotential $j_{3}: \mathbb{R} \rightarrow \mathbb{R}$ and its subdifferential are of the form

$$
j_{3}(r)=\left\{\begin{array}{ll}
0 & \text { if } r<0, \\
2 r^{2} & \text { if } 0 \leq r<1, \\
-\frac{1}{3} r^{3}+r^{2}+3 r-\frac{5}{3} & \text { if } 1 \leq r<3, \\
\frac{22}{3} & \text { if } r \geq 3,
\end{array} \quad \partial j_{3}(r)= \begin{cases}0 & \text { if } r<0, \\
4 r & \text { if } 0 \leq r<1, \\
-r^{2}+2 r+3 & \text { if } 1 \leq r<3, \\
0 & \text { if } r \geq 3 .\end{cases}\right.
$$

It is easy to check that the function $j_{3}$ satisfies $H\left(j_{3}\right)$. Furthermore, $j_{3}$ can be represented as the difference of convex functions, $j_{3}(r)=\varphi_{1}(r)-\varphi_{2}(r), r \in \mathbb{R}$ with

$$
\varphi_{1}(r)=\left\{\begin{array}{ll}
0 & \text { if } r<0, \\
2 r^{2} & \text { if } r \geq 0,
\end{array} \quad \varphi_{2}(r)= \begin{cases}0 & \text { if } r<1, \\
\frac{1}{3} r^{3}+r^{2}-3 r+\frac{5}{3} & \text { if } 1 \leq r<3, \\
2 r^{2}-\frac{22}{3} & \text { if } r \geq 3 .\end{cases}\right.
$$

Since $\varphi_{1}, \varphi_{2}$ are convex functions and $\partial \varphi_{1}$ is a singleton, from Lemma 14 of [29] we deduce that the function $-j_{3}$ is regular. Moreover, its subdifferential $\partial j_{3}$ is Lipschitz which implies that the condition $H\left(j_{3}\right)_{1}$ is fulfilled. 
In the second example, we consider the function $j_{3}: \mathbb{R} \rightarrow \mathbb{R}$ such that

$$
j_{3}(r)=\left\{\begin{array}{ll}
0 & \text { if } r<0, \\
r^{2} & \text { if } 0 \leq r<1, \\
\frac{1}{8} r^{4}-r^{3}+\frac{9}{4} r^{2}-\frac{3}{8} & \text { if } 1 \leq r<3, \\
3 & \text { if } r \geq 3,
\end{array} \quad \partial j_{3}(r)= \begin{cases}0 & \text { if } r<0, \\
2 r & \text { if } 0 \leq r<1, \\
\frac{1}{2} r^{3}-3 r^{2}+\frac{9}{2} r & \text { if } 1 \leq r<3, \\
0 & \text { if } r \geq 3\end{cases}\right.
$$

Similarly to the previous case, $j_{3}$ satisfies $H\left(j_{3}\right)_{1}$. It can be also represented as the difference of convex functions, $j_{3}(r)=\varphi_{1}(r)-\varphi_{2}(r), r \in \mathbb{R}$, where

$$
\varphi_{1}(r)=\left\{\begin{array}{ll}
0 & \text { if } r<0, \\
r^{2} & \text { if } r \geq 0,
\end{array} \quad \varphi_{2}(r)= \begin{cases}0 & \text { if } r<1, \\
-\frac{1}{8} r^{4}+r^{3}-\frac{5}{4} r^{2}+\frac{3}{8} & \text { if } 1 \leq r<3, \\
r^{2}-3 & \text { if } r \geq 3\end{cases}\right.
$$

Again, from the fact that $\varphi_{1}$ and $\varphi_{2}$ are convex functions and $\partial \varphi_{1}$ is a singleton, we conclude that $-j_{3}$ is regular.

We end this section with indications on specific applications of research on contact problems. It is of importance to provide various applications of the theoretical results to contact problems arising in real world. The applications concern the following areas.

Construction and exploitation of machines The understanding of contact problems are extremely important in various branches of engineering such as structural foundations, bearings, metal forming processes, drilling problems, the simulation of car crashes, the car braking system, contact of train wheels with the rails, a shoe with the floor, machine tools, bearings, motors, turbines, cooling of electronic devices, joints in mechanical devices, ski lubricants, and many more, cf., e.g., Andrews et al. [4], Chau et al. [5], Kuttler and Shillor [20, 21], Rochdi et al. [37] and Sofonea and Matei [43].

Biomechanics The applications concerns the medical field of arthoplasty where bonding between the bone implant and the tissue is of considerable importance. Artificial implants of knee and hip prostheses (both cemented and cement-less) demonstrate that the adhesion is important at the bone-implant interface. These applications are related to contact modeling and design of biomechanical parts like human joints, implants or teeth, cf. Panagiotopoulos [34], Rojek and Telega [38], Rojek et al. [39], Shillor et al. [41] and Sofonea et al. [42].

Plate tectonics and earthquakes predictions Results may be applicable to models with nonmonotone strain-stress laws in rock layers. Frictional contact between rocks are described by several models, cf. Dumont et al. [9], Ionescu et al. [12, 14], Ionescu and Nguyen [13], Ionescu and Paumier [15, 16] and Rabinowicz [36].

Medicine and biology Results are applicable to nonmonotone semipermeable membranes and walls (biological and artificial), cf. Duvaut and Lions [10]. In particular, contact problems for piezoelectric materials will continue to play a decisive role in the field of ultrasonic transducers for imaging applications, e.g., medical imaging (sonogram), nondestructive testing and high power applications (medical treatment, sonochemistry and industrial processing), cf. Shillor et al. [41], Sofonea et al. [42].

Acknowledgements Research partially supported by a Marie Curie International Research Staff Exchange Scheme Fellowship within the 7th European Community Framework Programme under Grant Agreement No. 295118. 
Open Access This article is distributed under the terms of the Creative Commons Attribution License which permits any use, distribution, and reproduction in any medium, provided the original author(s) and the source are credited.

\section{References}

1. Amassad, A., Fabre, C.: On the analysis of viscoplastic contact problem with time dependent Tresca's friction law. Electron. J. Math. Phys. Sci. 1, 47-71 (2002)

2. Amassad, A., Sofonea, M.: Analysis of a quasistatic viscoplastic problem involving Tresca friction law. Discrete Contin. Dyn. Syst. 4, 55-72 (1998)

3. Amassad, A., Sofonea, M.: Analysis of some nonlinear evolution systems arising in rate-type viscoplasticity. In: Chen, W., Hu, S. (eds.) Dynamical System and Differential Equations, an added volume to Discrete and Continuous Dynamical Systems, pp. 58-71 (1998)

4. Andrews, K.T., Shillor, M., Wright, S., Klarbring, A.: A dynamic viscoelastic contact problem with friction and wear. Int. J. Eng. Sci. 35, 1291-1309 (1997)

5. Chau, O., Han, W., Sofonea, M.: A dynamic frictional contact problem with normal damped response. Acta Appl. Math. 71, 159-178 (2002)

6. Clarke, F.H.: Optimization and Nonsmooth Analysis. Wiley-Interscience, New York (1983)

7. Denkowski, Z., Migórski, S., Papageorgiou, N.S.: An Introduction to Nonlinear Analysis: Theory. Kluwer Academic/Plenum, Boston (2003)

8. Denkowski, Z., Migórski, S., Papageorgiou, N.S.: An Introduction to Nonlinear Analysis: Applications. Kluwer Academic/Plenum, Boston (2003)

9. Dumont, Y., Goeleven, D., Rochdi, M., Kuttler, K.L., Shillor, M.: A dynamic model with friction and adhesion with applications to rocks. J. Math. Anal. Appl. 247, 87-109 (2000)

10. Duvaut, G., Lions, J.L.: Les Inéquations en Mécanique et en Physique. Dunod, Paris (1972)

11. Han, W., Sofonea, M.: Quasistatic Contact Problems in Viscoelasticity and Viscoplasticity. American Mathematical Society/International Press, Providence (2002)

12. Ionescu, I.R., Dascalu, C., Campillo, M.: Slip-weakening friction on a periodic system of faults: spectral analysis. Z. Angew. Math. Phys. 53, 980-995 (2002)

13. Ionescu, I.R., Nguyen, Q.L.: Dynamic contact problems with slip dependent friction in viscoelasticity. Int. J. Appl. Math. Comput. Sci. 12, 71-80 (2002)

14. Ionescu, I.R., Nguyen, Q.L., Wolf, S.: Slip-dependent friction in dynamic elasticity. Nonlinear Anal. 53, 375-390 (2003)

15. Ionescu, I.R., Paumier, J.-C.: On the contact problem with slip displacement dependent friction in elastostatics. Int. J. Eng. Sci. 34, 471-491 (1996)

16. Ionescu, I.R., Paumier, J.-C.: On the contact problem with slip rate dependent friction in elastodynamics. Eur. J. Mech. A, Solids 13, 555-568 (1994)

17. Kulig, A.: Hemivariational inequality approach to the dynamic viscoelastic contact problem with nonmonotone normal compliance and slip-dependent friction. Nonlinear Anal., Real World Appl. 9, 17411755 (2008)

18. Kulig, A.: Nonlinear evolution inclusions and hemivariational inequalities for nonsmooth problems in contact mechanics. PhD thesis, Jagiellonian University, Krakow, Poland (2010)

19. Kulig, A., Migórski, S.: Solvability and continuous dependence results for second order nonlinear evolution inclusions with a Volterra-type operator. Nonlinear Anal. Theory Methods Appl. (2012, in press)

20. Kuttler, K.L., Shillor, M.: Dynamic bilateral contact with discontinuous friction coefficient. Nonlinear Anal. 45, 309-327 (2001)

21. Kuttler, K.L., Shillor, M.: Dynamic contact with Signorini's condition and slip rate dependent friction. Electron. J. Differ. Equ. 83, 1-21 (2004)

22. Migórski, S.: On the existence of solutions for parabolic hemivariational inequalities. J. Comput. Appl. Math. 129, 77-87 (2001)

23. Migórski, S.: Dynamic hemivariational inequality modeling viscoelastic contact problem with normal damped response and friction. Appl. Anal. 84, 669-699 (2005)

24. Migórski, S.: Boundary hemivariational inequalities of hyperbolic type and applications. J. Glob. Optim. 31, 505-533 (2005)

25. Migórski, S.: Evolution hemivariational inequality for a class of dynamic viscoelastic nonmonotone frictional contact problems. Comput. Math. Appl. 52, 677-698 (2006)

26. Migórski, S., Ochal, A.: Hemivariational inequality for viscoelastic contact problem with slip dependent friction. Nonlinear Anal. 61, 135-161 (2005) 
27. Migórski, S., Ochal, A.: A unified approach to dynamic contact problems in viscoelasticity. J. Elast. 83, 247-275 (2006)

28. Migórski, S., Ochal, A.: Existence of solutions for second order evolution inclusions with application to mechanical contact problems. Optimization 55, 101-120 (2006)

29. Migórski, S., Ochal, A.: Dynamic bilateral contact problem for viscoelastic piezoelectric materials with adhesion. Nonlinear Anal. 69, 495-509 (2008)

30. Naniewicz, Z., Panagiotopoulos, P.D.: Mathematical Theory of Hemivariational Inequalities and Applications. Marcel Dekker, New York (1995)

31. Nečas, J., Hlaváček, I.: Mathematical Theory of Elastic and Elasto-plastic Bodies: An Introduction. Elsevier, Amsterdam (1981)

32. Ochal, A.: Existence results for evolution hemivariational inequalities of second order. Nonlinear Anal. 60, 1369-1391 (2005)

33. Panagiotopoulos, P.D.: Inequality Problems in Mechanics and Applications. Convex and Nonconvex Energy Functions. Birkhäuser, Basel (1985)

34. Panagiotopoulos, P.D.: Hemivariational Inequalities, Applications in Mechanics and Engineering. Springer, Berlin (1993)

35. Park, J.Y., Ha, T.G.: Existence of antiperiodic solutions for hemivariational inequalities. Nonlinear Anal. 68, 747-767 (2008)

36. Rabinowicz, E.: The nature of the static and kinetic coefficients of friction. J. Appl. Phys. 22, 1373-1379 (1951)

37. Rochdi, M., Shillor, M., Sofonea, M.: A quasistatic viscoelastic contact problem with normal compliance and friction. J. Elast. 51, 105-126 (1998)

38. Rojek, J., Telega, J.J.: Numerical simulation of bone-implant systems using a more realistic model of the contact interfaces with adhesion. J. Theor. Appl. Mech. 37, 659-686 (1999)

39. Rojek, J., Telega, J.J., Stupkiewicz, S.: Contact problems with friction adhesion and wear in orthopaedic biomechanics II: numerical implementation and application to implanted knee joints. J. Theor. Appl. Mech. 39, 679-706 (2001)

40. Selmani, M., Sofonea, M.: Viscoelastic frictionless contact problems with adhesion. J. Inequal. Appl. 2006, Article ID 36130 (2006), pp. 22

41. Shillor, M., Sofonea, M., Telega, J.J.: Models and Analysis of Quasistatic Contact. Springer, Berlin (2004)

42. Sofonea, M., Han, W., Shillor, M.: Analysis and Approximation of Contact Problems with Adhesion or Damage. Chapman \& Hall/CRC, Boca Raton (2006)

43. Sofonea, M., Matei, A.: Variational Inequalities with Applications. A Study of Antiplane Frictional Contact Problems. Advances in Mechanics and Mathematics. Springer, Berlin (2009)

44. Zeidler, E.: Nonlinear Functional Analysis and Applications, II A/B. Springer, New York (1990) 